Check for updates

Cite this: RSC Adv., 2018, 8, 36731

Received 18th July 2018

Accepted 23rd October 2018

DOI: $10.1039 / c 8 r a 06095 a$

rsc.li/rsc-advances

\section{Mechanisms of photoexcitation and photoionization in small water clusters $\uparrow$}

\author{
Parichart Suwannakham, Sermsiri Chaiwongwattana and Kritsana Sagarik (D)*
}

The mechanisms of photoexcitation and photoionization in small water clusters in gas phase, $\left(\mathrm{H}_{2} \mathrm{O}\right)_{n} ; n=$ $2-3$, are studied using the complete active-space second-order perturbation theory (CASPT2) with the aug-cc-pVDZ basis set. The present study characterizes for the first time the structures and energetics of common transition and intermediate complexes in the photoexcitation and photoionization mechanisms in the lowest singlet-excited state. The $a b$ initio results showed that the photoexcitation of the water monomer by a single photon can directly generate $[\mathrm{OH}]^{\circ}$ and $[\mathrm{H}]^{*}$ in their respective electronic-ground states, and a single photon with approximately the same energy can similarly lead to the photoexcitation and also to the photoionization in the water clusters. The $\mathrm{S}_{0} \rightarrow \mathrm{S}_{1}$ excitation leads to strong polarization of the $\mathrm{O}-\mathrm{H} \cdots \mathrm{O} \mathrm{H}$-bond and to the formation of the water dimer radical cation transition state complex $\left[\left(\mathrm{H}_{2} \mathrm{O}\right)_{2}\right]^{+\cdot}$, from which $[\mathrm{OH}]^{\cdot},[\mathrm{H}]^{\cdot}$, and $\left[\mathrm{H}_{3} \mathrm{O}\right]^{+\cdot}$ can be generated. These products are obtained from $\left[\left(\mathrm{H}_{2} \mathrm{O}\right)_{2}\right]^{+\cdot}$ by the direct dissociation of the $\mathrm{O}-\mathrm{H}$ bond upon photoexcitation and by proton transfer and the formation of a metastable charge-separated Rydberg-like $\mathrm{H}$-bond complex $\left(\left[\mathrm{H}_{3} \mathrm{O}\right]^{+\cdot} \cdots[\mathrm{OH}]^{*}\right)$ upon photoionization. The proposed mechanisms suggest that in the gas phase, the photoexcitation and photoionization processes are most likely bimolecular reactions, in which all the transition and intermediate charged species are more stabilized than in a unimolecular reaction. The theoretical results provide insights into the photoexcitation and photoionization mechanisms of molecular clusters and can be used as guidelines for further theoretical and experimental studies.

\section{Introduction}

The photochemistry of molecules in the Earth's atmosphere has been extensively studied in the past decades. The most important topic is the photolysis of small molecules, which leads to serious environmental problems. The photodissociation of water molecules is considered a prototypical reaction for theoretical and experimental studies of the $\mathrm{O}-\mathrm{H}$ bond cleavage in excited electronic states. ${ }^{1}$ Two different mechanisms for the photolysis of water molecules in gas phase were proposed, in which photoexcitation and photoionization represent the initial steps. $^{2}$ In the photoexcitation mechanism, the homolytic cleavage of the $\mathrm{O}-\mathrm{H}$ bond is the primary process, which forms $[\mathrm{H}]^{\cdot}$ and $[\mathrm{OH}]^{\cdot}$ (eqn (1)). The $[\mathrm{H}]^{\cdot}$ radical further reacts with another $\left[\mathrm{H}_{2} \mathrm{O}\right]$ molecule and generates $\left[\mathrm{H}_{3} \mathrm{O}\right]^{+}$and a hydrated electron ([e $\left.\left.{ }^{-}\right]^{\text {hyd }}\right)$ as the end products (eqn (2)):

$$
\begin{gathered}
{\left[\mathrm{H}_{2} \mathrm{O}\right] \stackrel{h v}{\longrightarrow}\left[\mathrm{H}_{2} \mathrm{O}\right]^{*} \rightarrow[\mathrm{H}]^{\cdot}+[\mathrm{OH}]^{\cdot}} \\
{[\mathrm{H}]^{\cdot}+\left[\mathrm{H}_{2} \mathrm{O}\right] \rightarrow\left[\mathrm{H}_{3} \mathrm{O}\right]^{+}+\left[\mathrm{e}^{-}\right]^{\text {hyd }}}
\end{gathered}
$$

School of Chemistry, Institute of Science, Suranaree University of Technology, Nakhon Ratchasima 30000, Thailand. E-mail: kritsana@sut.ac.th; Fax: +6644 224635; Tel: +6644224635

$\dagger$ Electronic supplementary information (ESI) available. See DOI: 10.1039/c8ra06095a
In the photoionization mechanism, the ionic products $\left[\mathrm{H}_{2} \mathrm{O}\right]^{+}$and $\left[\mathrm{e}^{-}\right]^{\text {hyd }}$ are primarily generated (eqn (3)), followed by a bimolecular reaction between $\left[\mathrm{H}_{2} \mathrm{O}\right]^{+}$and $\left[\mathrm{H}_{2} \mathrm{O}\right]$ to produce $\left[\mathrm{H}_{3} \mathrm{O}\right]^{+}$and $[\mathrm{OH}]^{\bullet}($ eqn $(4))$ :

$$
\begin{gathered}
{\left[\mathrm{H}_{2} \mathrm{O}\right] \stackrel{h v}{\longrightarrow}\left[\mathrm{H}_{2} \mathrm{O}\right]^{+}+\left[\mathrm{e}^{-}\right]^{\text {hyd }}} \\
{\left[\mathrm{H}_{2} \mathrm{O}\right]^{+}+\left[\mathrm{H}_{2} \mathrm{O}\right] \rightarrow\left[\mathrm{H}_{3} \mathrm{O}\right]^{+}+[\mathrm{OH}]^{\cdot}}
\end{gathered}
$$

Although the total reactions are the same, starting from a single water molecule and ending with $\left[\mathrm{H}_{3} \mathrm{O}\right]^{+},[\mathrm{OH}]^{\circ}$, and $\left[\mathrm{e}^{-}\right]^{\text {hyd }}$, the photochemistry of photoexcitation and photoionization are completely different. $A b$ initio calculations have showed that photoexcitation (eqn (1)) dominates in gas phase and corresponds to the HOMO-LUMO excitation $(7.5 \mathrm{eV}){ }^{3}$ whereas the photoionization of an isolated water molecule (eqn (3)) requires considerably higher energy $(12.6 \mathrm{eV}){ }^{4}$ The former is in accordance with UV spectroscopic experiments on single water molecules in gas phase, which indicated that the valence character of the lowest energy band of the electronic spectrum is broad, ranging from 6.8 to $8.2 \mathrm{eV}(182-165 \mathrm{~nm}) .{ }^{5}$ Moreover, the maximum at $7.4 \mathrm{eV}(168 \mathrm{~nm})^{6}$ is associated with the direct photodissociation of the $\mathrm{O}-\mathrm{H}$ bond into $[\mathrm{OH}]^{\cdot}$ and $[\mathrm{H}]^{\cdot}$ in the electronic ground state. ${ }^{7}$ 
The photoexcitation and photoionization of small water clusters, $\left(\mathrm{H}_{2} \mathrm{O}\right)_{n} ; n=2-3$, were studied using various quantum chemical methods and $a b$ initio molecular dynamics (AIMD) simulations. ${ }^{2}$ The AIMD results based on the complete activespace self-consistent field (CASSCF) method showed that the photoexcitation and photoionization processes are complementary in the singlet-excited state, and the photoionization process, which involves structural changes and a proton transfer in hydrogen bond (H-bond), is slower owing to the lack of $[\mathrm{H}]^{\cdot}$ radicals. At the beginning of the AIMD simulations, the transferring proton possesses a high kinetic energy, and the Eigen and Zundel complexes compete; after $500 \mathrm{fs}$, proton transfer takes place, leading to the formation of $\left[\mathrm{H}_{3} \mathrm{O}\right]^{+}$and $[\mathrm{OH}]^{\circ}$.

Water molecules in the singlet- and triplet-excited states were theoretically studied using the extended multistate complete active-space with second-order perturbation theory (MS-CASPT2). ${ }^{7}$ The analysis of the valence and Rydberg characters of the lowest-lying $\left(1^{1} \mathrm{~B}_{1}\right)$ state of different geometries showed that the $3 \mathrm{~s}$-Rydberg character dominates the groundstate equilibrium structure, which is transformed into a valence state upon $1 \mathrm{~b}_{1} \rightarrow 4 \mathrm{a}_{1}$ excitation via an extension of both the $\mathrm{O}-\mathrm{H}$ bonds ( $C_{2 \mathrm{v}}$ symmetry). Low-lying excited states of $\mathrm{H}_{2} \mathrm{O}, \mathrm{CH}_{3} \mathrm{OH}$, and dimethyl ether $\left(\mathrm{C}_{2} \mathrm{H}_{6} \mathrm{O}\right)$ were studied using the Hartree-Fock method and improved virtual orbital (IVO) calculations. $^{8}$ The results indicated that the low-lying excited states of these molecules are represented by the excitation of an oxygen lone-pair to a 3s or 3p Rydberg-like orbital located on the oxygen atom; moreover, due to the interaction between the electron-deficient hydrogen atoms, the Rydberg orbitals in water are more stable (i.e., have a lower excitation energy) than in $\mathrm{CH}_{3} \mathrm{OH}$ and $\mathrm{C}_{2} \mathrm{H}_{6} \mathrm{O}$.

The spectral signature of the hydrated electron in aqueous solutions was studied using $a b$ initio calculations and density functional theory (DFT), in which the electronic ground state and the lowest singlet-excited state of the water dimer and the microsolvation of a hydronium radical (with up to two hydration shells) were primarily investigated. ${ }^{9}$ Because the potential energy curve as a function of the $\mathrm{O}-\mathrm{H}$ coordinate obtained from CASPT2 calculations is barrierless, the $S_{0} \rightarrow S_{1}$ excitation of the water dimer leads to a concerted electron-hydrogen transfer in the $\mathrm{H}$-bond and a separation of $\left[\mathrm{H}_{3} \mathrm{O}\right]^{+}$and $[\mathrm{OH}]^{\circ}$. The hydrated hydronium ion is represented by a charge-separated complex, which is characterized by $\mathrm{a}_{3} \mathrm{O}^{+}$ion and a localized-electron cloud.

The barrierless potential energy curve obtained contrasts with previous CASSCF results, in which the $S_{1}$ potential energy curve obtained based on a freeze-scan method exhibited a low energy barrier associated with proton transfer in the water dimer and the formation of the $\mathrm{H}_{3} \mathrm{O}^{+}$cation. ${ }^{\mathbf{1 0}}$ This discrepancy was attributed to the lack of diffuse basis functions, which are essential for the calculation of $\sigma^{*}$ Rydberg orbitals, and the use of the freeze-scan method, in which the $\mathrm{O}-\mathrm{H}$ potential energy curve is constructed by constraining all the structural parameters to their equilibrium values. ${ }^{9}$

In this work, the mechanisms of photodissociation of the water molecule and small water clusters in gas phase, $\left(\mathrm{H}_{2} \mathrm{O}\right)_{n} ; n$
$=2-3$, were studied using CASPT2 with the aug-cc-pVDZ basis set. In contrast to the previous theoretical studies, which considered the photoexcitation and photoionization mechanisms separately, ${ }^{2,9-11}$ the present study focused on common intermediate and transition state complexes in both processes in the lowest-singlet excited $\left(S_{1}\right)$ state. The structures and energetics of the elementary steps as well as the relaxations of the structures of the excited molecules in the photodissociation processes were studied using the $S_{1}$ potential energy curves as a function of the degrees of freedom. The effects of the cluster size were studied and discussed in comparison with available theoretical and experimental data.

\section{Computational methods}

Our theoretical studies on protonated H-bonded systems showed that the dynamics and mechanisms of proton transfer could be reasonably studied based on the concept of presolvation. ${ }^{\mathbf{1 2 - 1 4}}$ In this approach, the smallest, most active intermediate complex in a systematically selected presolvation model is used as a representative system and studied in detail. ${ }^{15-18}$ For example, for protonated water systems, ${ }^{\mathbf{1 6}, 17}$ the rate-determining step of proton transfer in the electronic ground state is characterized by the interconversion between the close-contact $\left(\mathrm{O}-\mathrm{H}^{+} \cdots \mathrm{O}\right)$ and shared-proton $\left(\mathrm{O} \cdots \mathrm{H}^{+} \cdots \mathrm{O}\right)$ structures in $\mathrm{H}_{5} \mathrm{O}_{2}{ }^{+}$. Moreover, the smallest, most active intermediate complex for photoacid dissociation in microhydrated hydroxylamine clusters in the $\mathrm{S}_{1}$ state is the charge-separated Rydberg-like H-bond complex $\left[\mathrm{NH}_{2} \mathrm{O}\right]^{\bullet} \cdots\left[\mathrm{H}_{3} \mathrm{O}\right]^{+\bullet} \cdot{ }^{19}$ Therefore, to study the photoexcitation and photoionization processes in gas phase (eqn (1)-(4)), the water molecule and small water clusters, $\left(\mathrm{H}_{2} \mathrm{O}\right)_{n} ; n=2-3$, were used as model systems. ${ }^{2}$

To simplify the discussion, the water molecule and small water clusters were labeled with a three-character code, $\mathrm{G} n$ - $[m]$ and $\mathrm{E} n-[m]$; where $\mathrm{G}$ is the structure of the water cluster in the $\mathrm{S}_{0}$ state; $\mathrm{E}$ is the structure of the water cluster in the $\mathrm{S}_{1}$ state; $n$ is the number of water molecules. The structures with the same number of water molecules are differentiated by $m$. For example, G2-[1] and E2-[1] are water dimers with similar structures in the $S_{0}$ and $S_{1}$ state, respectively, whereas E2-[1] and E2-[2] are different structures in the $S_{1}$ state.

\section{Quantum chemical calculations}

$A b$ initio calculations in the $\mathrm{S}_{1}$ state were performed using the CASPT2 method, which has been successfully used in the past decades and widely accepted as a standard method for excitedstate calculations. ${ }^{20}$ Because photochemical reactions in water clusters involve covalent bond breaking and/or forming, at least two configurations representing close-shell and open-shell configurations must be included in the calculations; ${ }^{2}$ the analysis of the valence and Rydberg character of the lowest-lying state showed that the 3s-Rydberg character dominates the ground-state equilibrium structure, which is transformed into a valence state upon $S_{0} \rightarrow S_{1}$ excitation via an extension of the O-H bond. ${ }^{7}$ 
Table 1 The CASPT2 methods, the number of CSF considered ( $N$ e-internal) and those generated by the CASPT2 method ( $N-1$ and $N-2$ einternal) used in the calculations of $\left(\mathrm{H}_{2} \mathrm{O}\right)_{n} ; n=1-3 . n=$ number of active electrons; $m=$ number of active orbitals; $E^{\mathrm{Ex}}=$ vertical excitation energies in eV

\section{Number of CSFs}

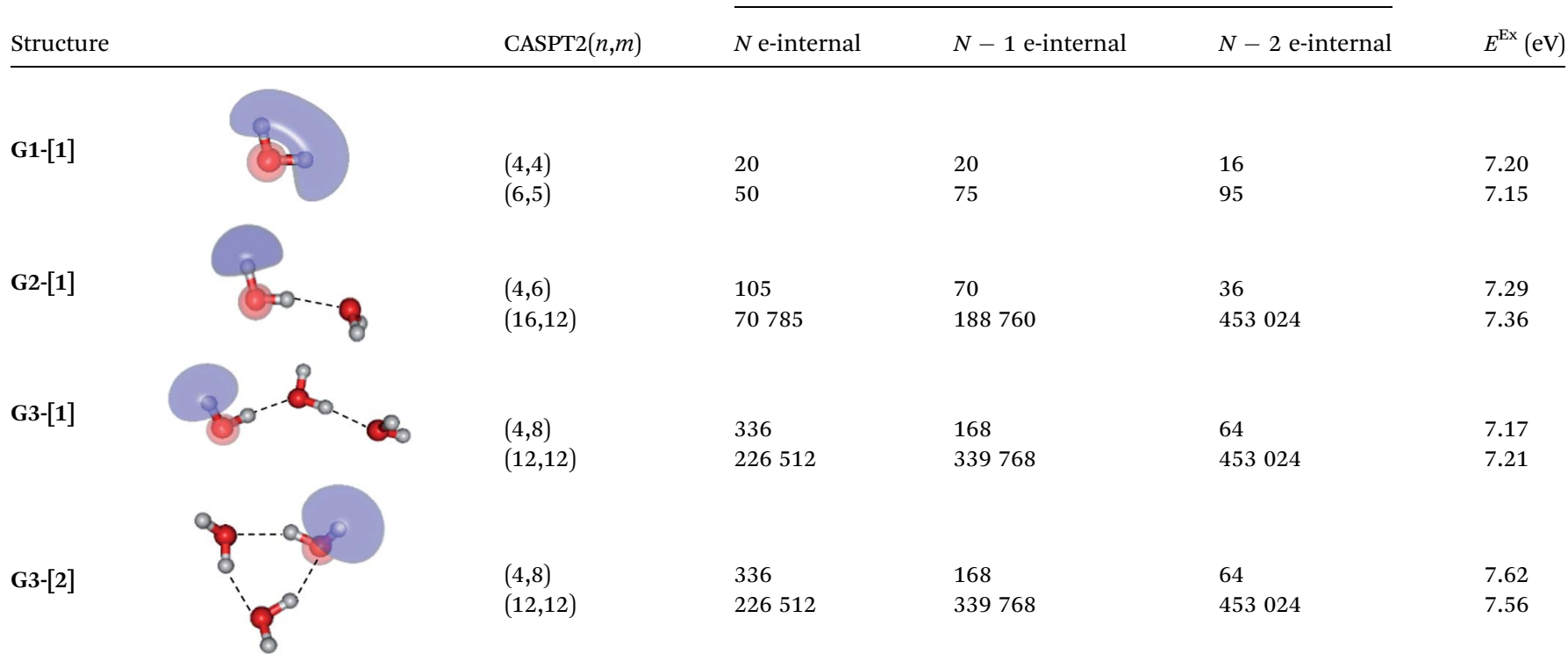

The $\left(\mathrm{H}_{2} \mathrm{O}\right)_{n} ; n=2-3$ model clusters are considered too large for the CASPT2 geometry optimizations and calculations of the $S_{1}$ relax-scan potential energy curves, in which the energy gradients with respect to degrees of freedom are extensively computed. Therefore, to optimize the computational resources, the aug-cc-pVDZ basis set was used. Moreover, due to the fast convergence of the excitation energy, augmented basis sets with diffusion functions are recommended for singlet-state calculations. ${ }^{21}$ In addition, as the Hartree-Fock method ${ }^{8}$ and preliminary CASPT2 calculations showed that a single photon only excites one oxygen atom in the water molecule, the accuracy and CPU time were further compromised by assigning only four lone-pair electrons of one oxygen atom in the active orbitals while the remaining electrons were included in doubly occupied

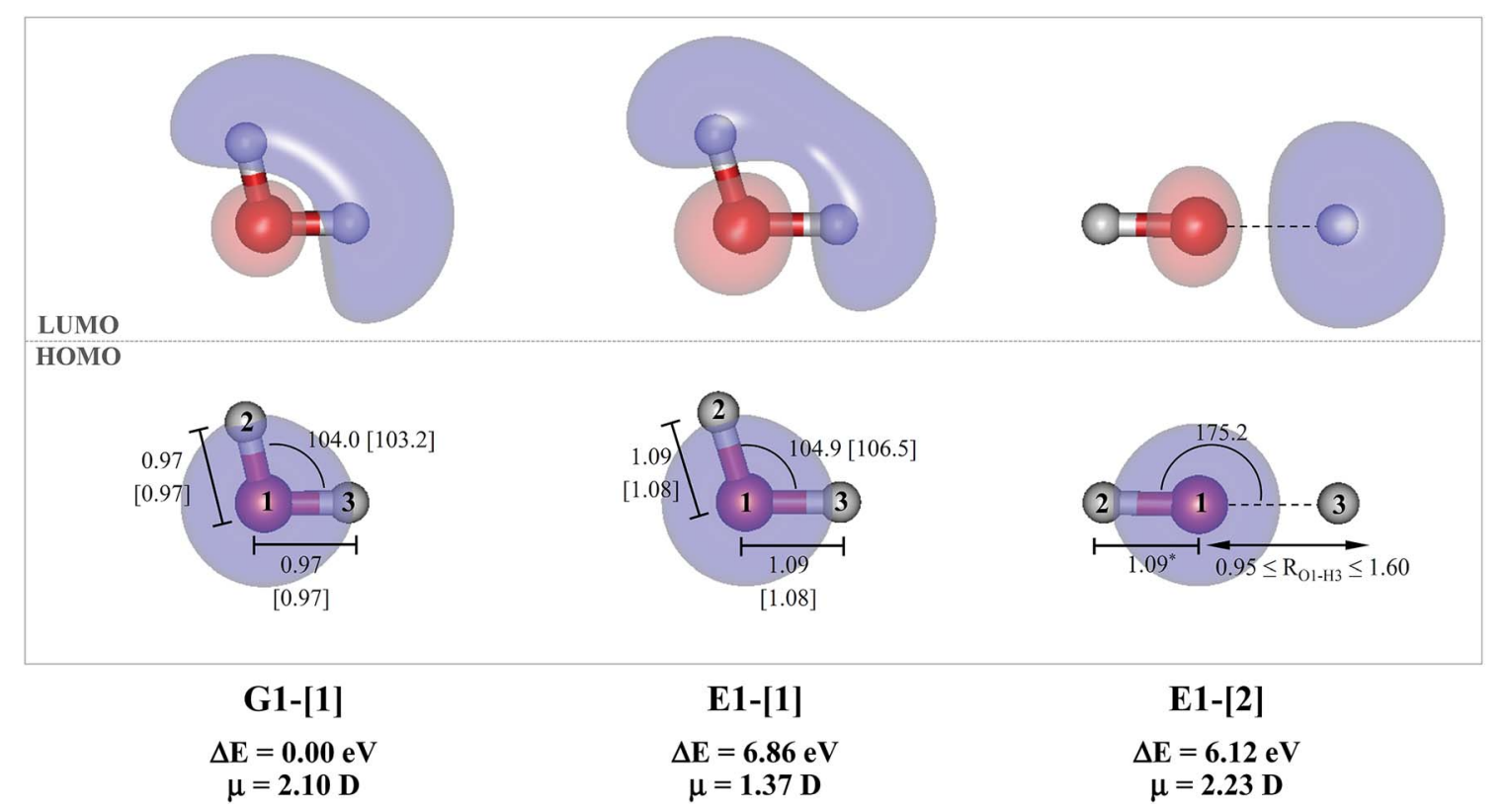

Fig. 1 G1-[1] and E1-[1] = equilibrium structures of the water monomer in $C_{2 v}$ symmetry obtained from CASPT2 $(4,4)$ calculations in the $S_{0}$ and $S_{1}$ states, respectively; E1-[2] = partially-constrained water monomer $\left(R_{\mathrm{O}-\mathrm{H}}=1.09 \AA\right)$ used in the calculations of the $\mathrm{S}_{1}$ relax-scan potential energy curve for the $\mathrm{O}-\mathrm{H}$ dissociation. Distances and angles are in $\AA$ and degree, respectively. The values in square brackets were obtained from CASPT2 $(6,5)$ geometry optimizations. The value of the HOMO and LUMO isosurfaces is $0.065 . \Delta E=$ relative energies with respect to the total energy of G1-[1]; $\mu=$ dipole moment. 


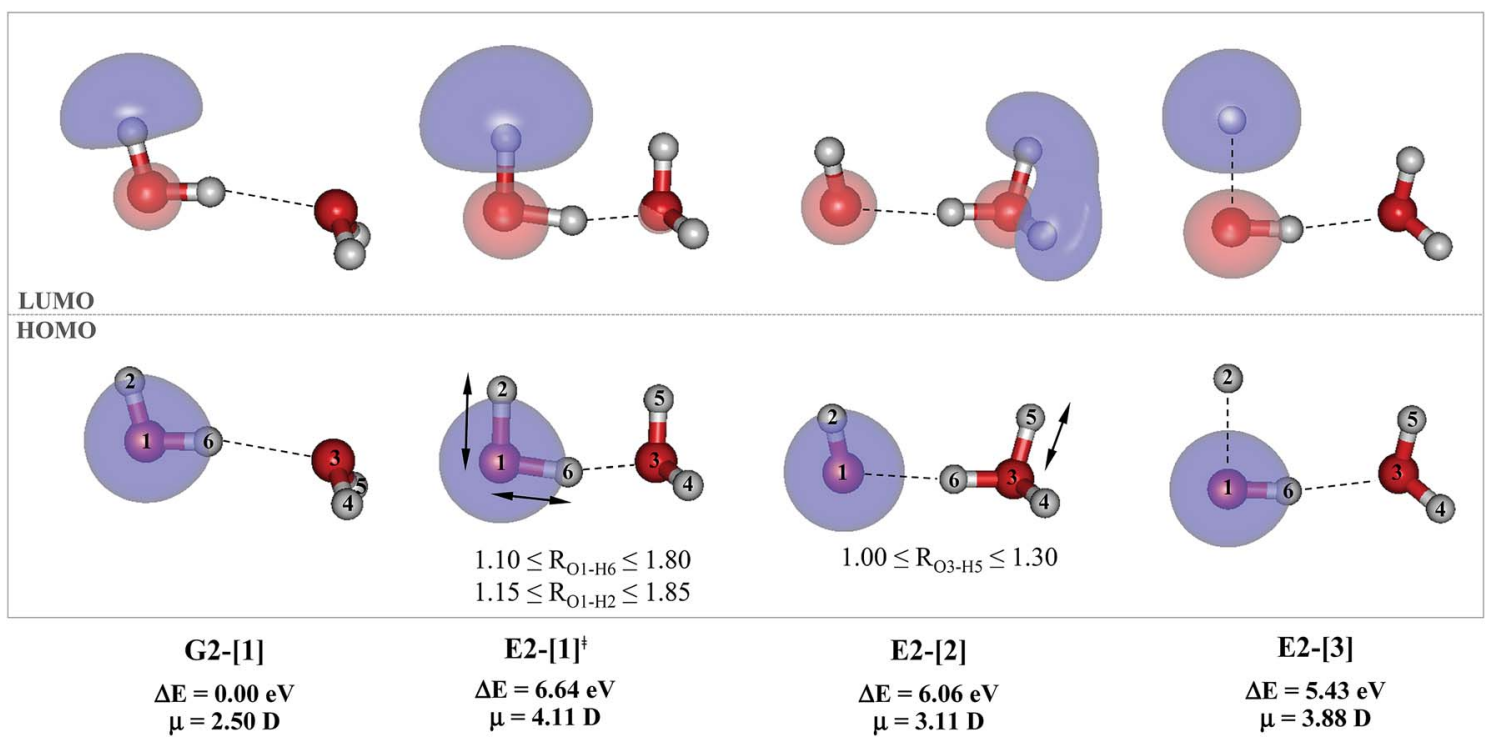

Fig. 2 Structures of the water dimer obtained from CASPT2 $(4,6)$ calculations in the $\mathrm{S}_{0}$ and $\mathrm{S}_{1}$ states; $\mathrm{G} 2$ - $[1]=$ equilibrium structure in the $\mathrm{S}_{0}$ state; $\mathrm{E} 2-[1]^{*}=$ transition structure in the $\mathrm{S}_{1}$ state; $\mathrm{E} 2-[2]=$ equilibrium structure in the $\mathrm{S}_{1}$ state; $\mathrm{E} 2-[3]=\mathrm{O}-\mathrm{H}$ dissociated structure in the $\mathrm{S}_{1}$ state. Distances are in $\AA$ and the value of the $\mathrm{HOMO}$ and LUMO isosurfaces is 0.065 . $\Delta E=$ Relative energies with respect to the total energy of structure G2-[1]; $\mu=$ dipole moment.

orbitals. For the water monomer, the four lone-pair electrons $(n$ $=4)$ were assigned in four active orbitals $(m=4)$, whereas the remaining six electrons occupied three closed-shell orbitals (close $=3$ ), abbreviated CASPT2 $(4,4)$. For the water dimer, the same number of electrons $(n=4)$ were assigned in six active orbitals $(m=6)$, whereas the remaining sixteen electrons occupied eight closed-shell orbitals (close $=8$ ), abbreviated CASPT2 $(4,6)$. Based on the same approach, CASPT2 $(4,8)$ was used in the water trimer calculations.

To ensure that CASPT2 calculations with restricted number of active electrons $(n=4)$ and active orbitals did not lead in serious errors in the structural and energetic results, CASPT2 calculations with larger active spaces $($ close $=2)$ were performed on the water monomer and dimer, CASPT2 $(6,5)$ and $(16,12)$, respectively. For the water trimer, due to excessive number of configuration state functions (CSF) and the limitation of our computer resources, CASPT2 calculations with close $=2$ were not possible. Therefore, only twelve lone-pair electrons of the three oxygen atoms were assigned in twelve active orbitals, CASPT2 $(12,12)$ with close $=9$. The information on the CASPT2 methods used in this work are summarized in Table 1. To characterize the $S_{0} \rightarrow S_{1}$ excited species, the spatial distributions of the HOMO and LUMO of the water clusters were computed and illustrated with the same value of the isosurface. All of the CASPT2 calculations were performed using a statespecific method included in the MOLPRO software package. ${ }^{22,23}$ The Werner-Meyer-Knowles non-linear optimization method ${ }^{24-26}$ was used in the orbital/state optimizations.

\section{Equilibrium structures and potential energy curves}

The water monomer and clusters in the $S_{0}$ state were fully optimized in the $C_{1}$ symmetry. To obtain fundamental information on the mechanisms of photoexcitation and photoionization in the $S_{1}$ state, the equilibrium and transition structures of the water monomer and dimer were calculated using the quadratic steepest decent (QSD) method included in the MOLPRO software package; the QSD method for transition state calculations is based on the image Hessian method of Sun and Ruedenberg. ${ }^{27}$ To study the structural changes along the photodissociation paths, the $\mathrm{S}_{1}$ potential energy curves as a function of the $\mathrm{O}-\mathrm{H}$ coordinates were constructed using a relax-scan method and the $S_{0}$ potential energy curves were calculated at the same geometries. ${ }^{9}$

For the water monomer in the $\mathrm{S}_{1}$ state, due to the predominance of the homolytic cleavage of an $\mathrm{O}-\mathrm{H}$ bond into $[\mathrm{OH}]^{\cdot}$ and $[\mathrm{H}]^{\circ}$, the $\mathrm{S}_{1}$ relax-scan potential energy curve was constructed by constraining the $\mathrm{O}-\mathrm{H}$ bond $\left(R_{\mathrm{O}-\mathrm{H}}=1.09 \AA\right)$ while varying the other bond distance over the range of 0.95 to $1.60 \AA$ (structure E1-[2] in Fig. 1). It should be noted that although the $S_{1}$ relaxscan potential energy curve with a constrained $\mathrm{O}-\mathrm{H}$ bond is not realistic, we would like to obtain complete information to confirm that the $S_{1}$ potential energy curves obtained from CASPT2 calculations with restricted (CASPT2 $(4,4)$ ) and extended complete active spaces (CASPT2(6,5)) lead to the same results for the water monomer.

For the water dimer, based on literature survey and preliminary CASPT2 calculations in the $\mathrm{S}_{0}$ and $\mathrm{S}_{1}$ states, at least four structures (Fig. 2) were hypothesized to be involved in the photoexcitation and photoionization mechanisms; G2-[1] is the equilibrium structure in the $S_{0}$ state; E2-[1] $]^{\ddagger}$ and E2-[2] are the transition and equilibrium structures in the $\mathrm{S}_{1}$ state, respectively; and E2-[3] is the direct $\mathrm{O}-\mathrm{H}$ dissociation structure in the $\mathrm{S}_{1}$ state. To calculate the $\mathrm{S}_{1}$ relax-scan potential energy curve for the direct $\mathrm{O}-\mathrm{H}$ dissociation (E2-[3]), the $\mathrm{O}_{1}-\mathrm{H}_{2}$ coordinate of the transition structure (E2-[1] $]^{\ddagger}$ was varied over the range of 1.15- 
Table 2 Structures of the water monomer, main electronic configurations and corresponding $\mathrm{Cl}$ coefficients (cut-off $=0.05)$ obtained from CASPT2 $(4,4)$ and $(6,5)$ calculations $^{a}$

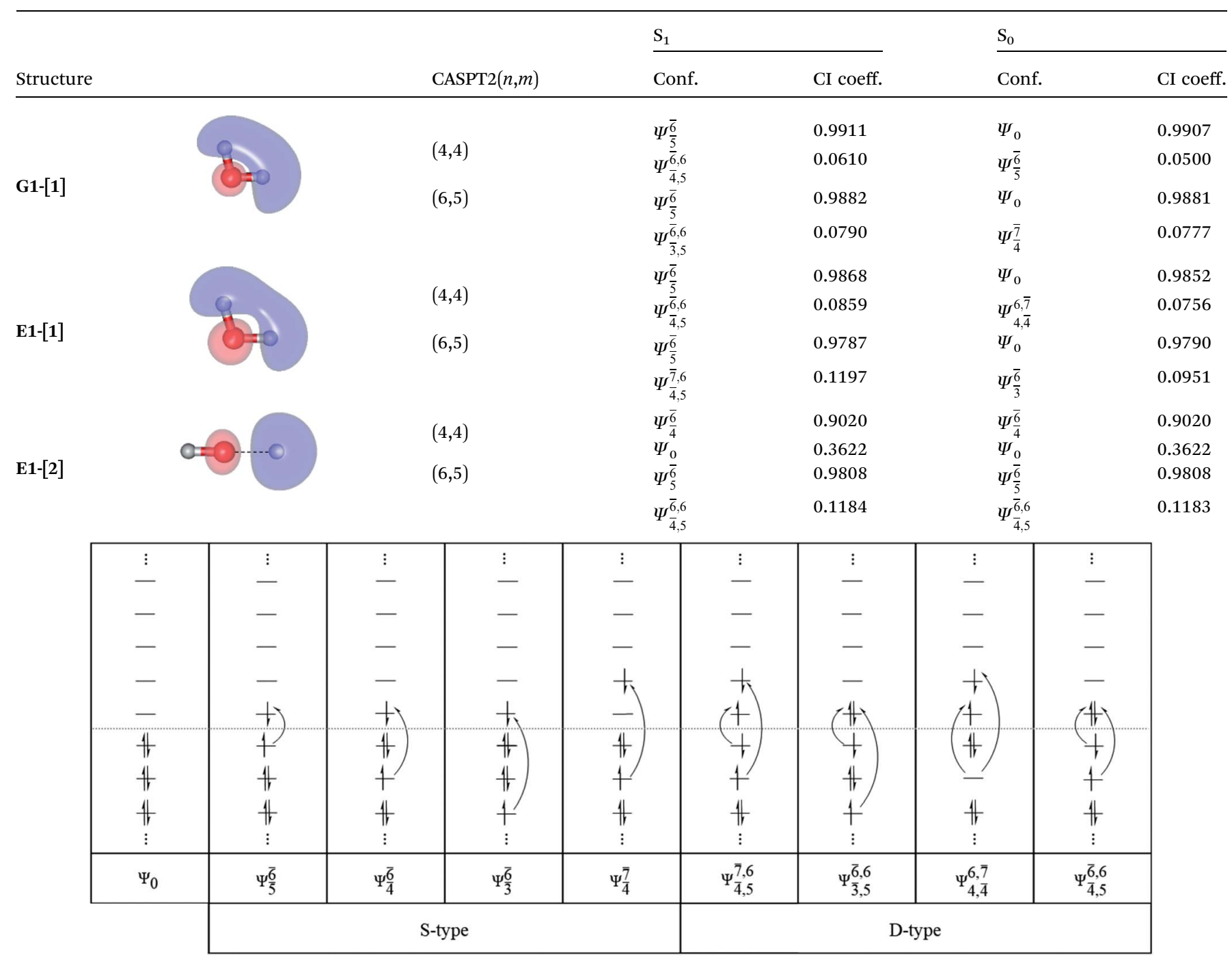

${ }^{a} \Psi_{a}^{r}$ and $C_{a}^{r}=a \rightarrow r$ singly excited determinant (S-type) and CI coefficient and; $\Psi_{a, b}^{r, s}$ and $C_{a, b}^{r, s}=a \rightarrow r$ and $b \rightarrow s$ doubly excited determinant (D-type) and CI coefficient; the indices a and $\mathrm{b}$, and $\mathrm{r}$ and s label occupied and virtual or unoccupied spin orbitals, respectively; a bar or lack of a bar is to denote beta $(\beta)$ and alpha $(\alpha)$ spin orbitals, respectively.

$1.85 \AA$ A. In the calculation of the $\mathrm{S}_{1}$ relax-scan potential energy curve for the hydrogen/proton transfer in the $\mathrm{H}$-bond, the $\mathrm{O}_{1}-$ $\mathrm{H}_{6}$ coordinate of structure E2-[1] $]^{*}$ was varied over the range of 1.10-1.80 A. Similarly, to construct the $\mathrm{S}_{1}$ relax-scan potential energy curve for the hydrogen/proton transfer from the $\mathrm{H}_{3} \mathrm{O}$ moiety of $\mathbf{E 2}$-[2], the $\mathrm{O}_{3}-\mathrm{H}_{5}$ coordinate was varied over the range of 1.00-1.30 A. All the $S_{1}$ relax-scan potential energy curves were computed with a step of $\Delta R_{\mathrm{O}-\mathrm{H}}=0.05 \AA$, and the coordinates on the $S_{1}$ relax-scan potential energy curves were used in the calculations of the $S_{0}$ potential energy curves.

Based on the same approach, the equilibrium and transition structures as well as the $S_{1}$ relax-scan potential energy curves for the photodissociation of the water trimer were computed using CASPT2 calculations. These calculations were performed to study the effect of the cluster size, especially the extension of the
$\mathrm{O}-\mathrm{H} \cdots \mathrm{O}$ chain, on the structure and energetics of the transition and intermediate complexes.

\section{Results and discussion}

The structure parameters and energies obtained from CASPT2 calculations with restricted complete active spaces are listed in Table S1.† The discussion will be focused primarily on the equilibrium and transition structures as well as on the relaxscan potential energy curves of the $S_{0}$ and $S_{1}$ states of the water monomer and dimer. Because the structures, energetics and $S_{1}$ relax-scan potential energy curves obtained from CASPT2 calculations with restricted and extended complete active spaces were not significantly different, the discussion will be made based on the CASPT2 results with restricted complete 
Table 3 Structures of the water dimer, main electronic configurations and corresponding $\mathrm{Cl}$ coefficients (cut-off $=0.05)$ obtained from CASPT2 $(4,6)$ and $(16,12)$ calculations $^{a}$

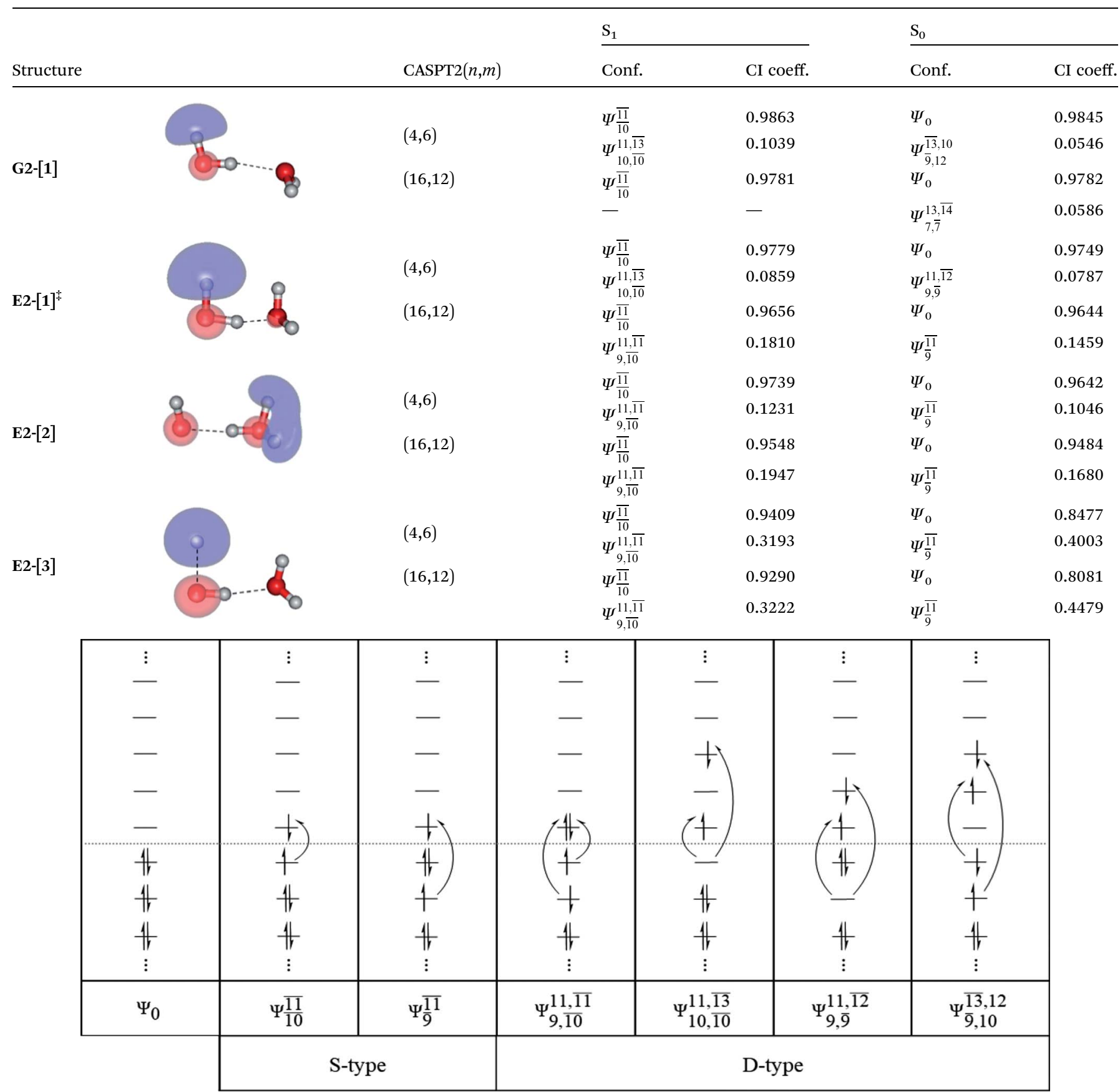

${ }^{a} \Psi_{a}^{r}$ and $C_{a}^{r}=a \rightarrow r$ singly excited determinant (S-type) and CI coefficient and; $\Psi_{a, b}^{r, s}$ and $C_{a, b}^{r, s}=a \rightarrow r$ and $b \rightarrow s$ doubly excited determinant (D-type) and CI coefficient; the indices $a$ and $b$, and $r$ and $s$ label occupied and virtual or unoccupied spin orbitals, respectively; a bar or lack of a bar is to denote beta $(\beta)$ and alpha $(\alpha)$ spin orbitals, respectively.

active spaces. The photoexcitation and photoionization mechanisms proposed based on the results of the water monomer and dimer were confirmed using the results of the water trimer. The multireference characters of the wave functions of the water monomer, dimer and trimer in the $S_{0}$ and $S_{1}$ states are discussed based on the main electronic configurations and the corresponding configuration interaction (CI) coefficients in Tables 2-4, respectively.

\section{Equilibrium structures of the water monomer and dimer}

The equilibrium structures of a single water molecule in the $S_{0}$ and $S_{1}$ state are shown with the spatial distributions of the 
Table 4 Structures of the water trimer, main electronic configurations and corresponding $\mathrm{Cl}$ coefficients (cut-off $=0.05$ ) obtained from CASPT2 $(4,8)$ and $(12,12)$ calculations $^{a}$

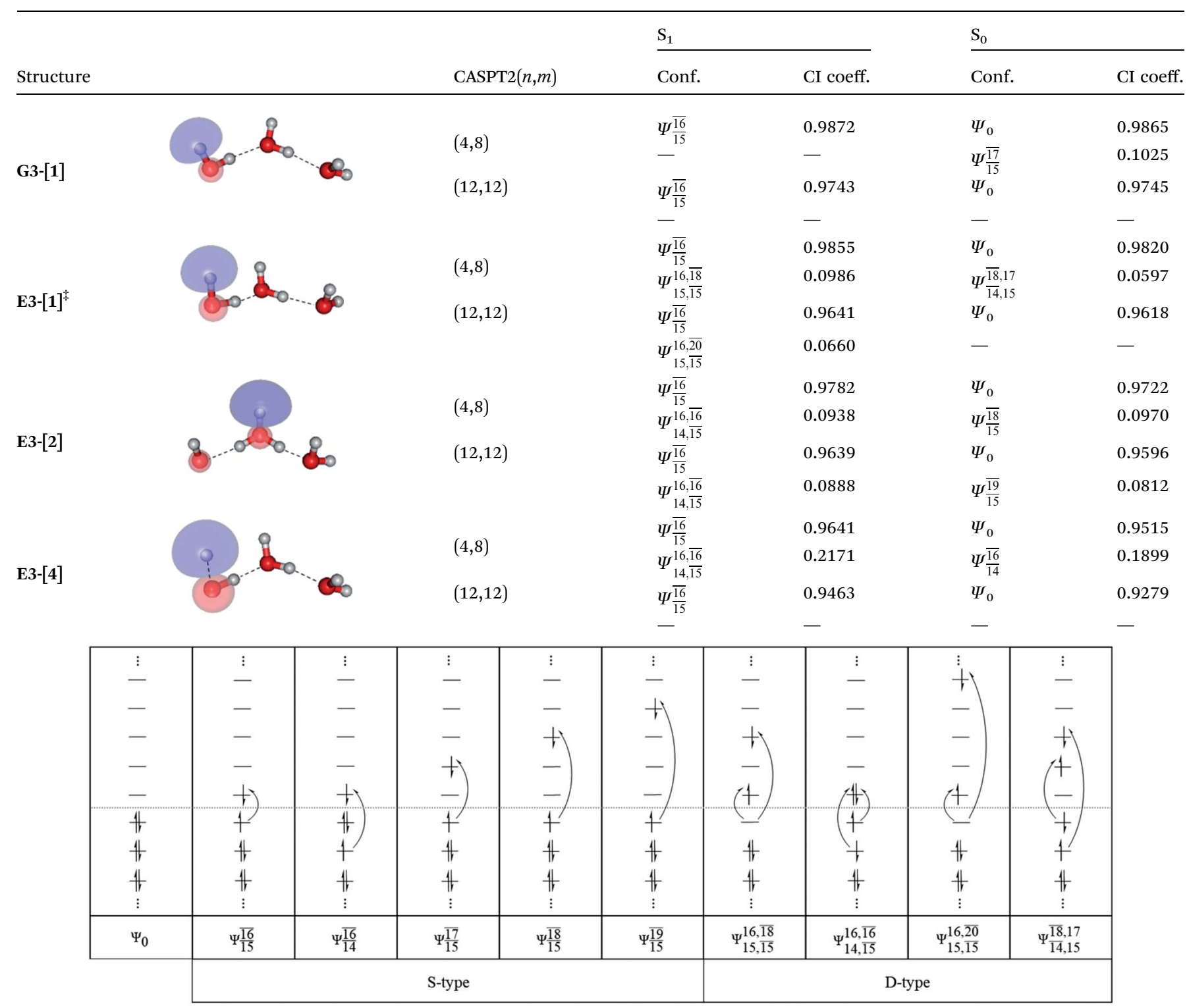

${ }^{a} \Psi_{a}^{r}$ and $C_{a}^{r}=a \rightarrow r$ singly excited determinant (S-type) and CI coefficient and; $\Psi_{a, b}^{r, s}$ and $C_{a, b}^{r, s}=a \rightarrow r$ and $b \rightarrow s$ doubly excited determinant (D-type) and CI coefficient; the indices $a$ and $b$, and $r$ and $s$ label occupied and virtual or unoccupied spin orbitals, respectively; a bar or lack of a bar is to denote beta $(\beta)$ and alpha $(\alpha)$ spin orbitals, respectively.

HOMO and LUMO in Fig. 1. Following the CASPT2 $(4,4)$ geometry optimizations, the equilibrium structure of the water monomer in the $\mathrm{S}_{0}$ state (G1-[1]) is characterized by $R_{\mathrm{O}-\mathrm{H}}=0.97$ $\AA$ and $\angle \mathrm{HOH}=104.0^{\circ}$ with $\mathrm{a} \mathrm{S}_{0} \rightarrow \mathrm{S}_{1}$ vertical excitation energy of $E^{\mathrm{Ex}}=7.20 \mathrm{eV}$, which is in excellent agreement with the sharp UV absorption peak observed in the range of 6.9 to $7.5 \mathrm{eV}^{5,6}$ Similar results were obtained from the CASPT2 $(6,5)$ geometry optimizations: $R_{\mathrm{O}-\mathrm{H}}=0.97 \AA, \angle \mathrm{HOH}=103.2^{\circ}, E^{\mathrm{Ex}}=7.15 \mathrm{eV}$. In the $\mathrm{S}_{1}$ state, the CASPT2 $(4,4)$ geometry optimizations with $C_{1}$ symmetry led to the direct dissociation of an $\mathrm{O}-\mathrm{H}$ bond (structure E1-[2] in Fig. 1), whereas the equilibrium structure with the symmetry constrained in the $C_{2 \mathrm{v}}$ point group exhibited
$R_{\mathrm{O}-\mathrm{H}}=1.09 \AA$ and $\angle \mathrm{HOH}=104.9^{\circ}($ E1-[1] $)$. The latter values are comparable with the results obtained from the CASPT2 $(6,5)$ geometry optimizations: $R_{\mathrm{O}-\mathrm{H}}=1.08 \AA$ and $\angle \mathrm{HOH}=106.5^{\circ}$. The spatial distributions of the LUMO in Fig. 1 suggest a predominance of the charge-separated Rydberg-like complex in the $S_{1}$ state (structures G1-[1] and E1-[1] with $C_{2 \mathrm{v}}$ symmetry) and a homolytic cleavage of the $\mathrm{O}-\mathrm{H}$ bond to yield $[\mathrm{H}]^{\circ}$ and $[\mathrm{OH}]^{\cdot}$ (structure E1-[2], with the HOMO-LUMO excitation energy of $10.38 \mathrm{eV})$.

The characteristic structures of the water dimer in the $S_{0}$ and $\mathrm{S}_{1}$ states obtained from CASPT2 $(4,6)$ calculations are included in Fig. 2. The equilibrium structure of the water dimer in the 


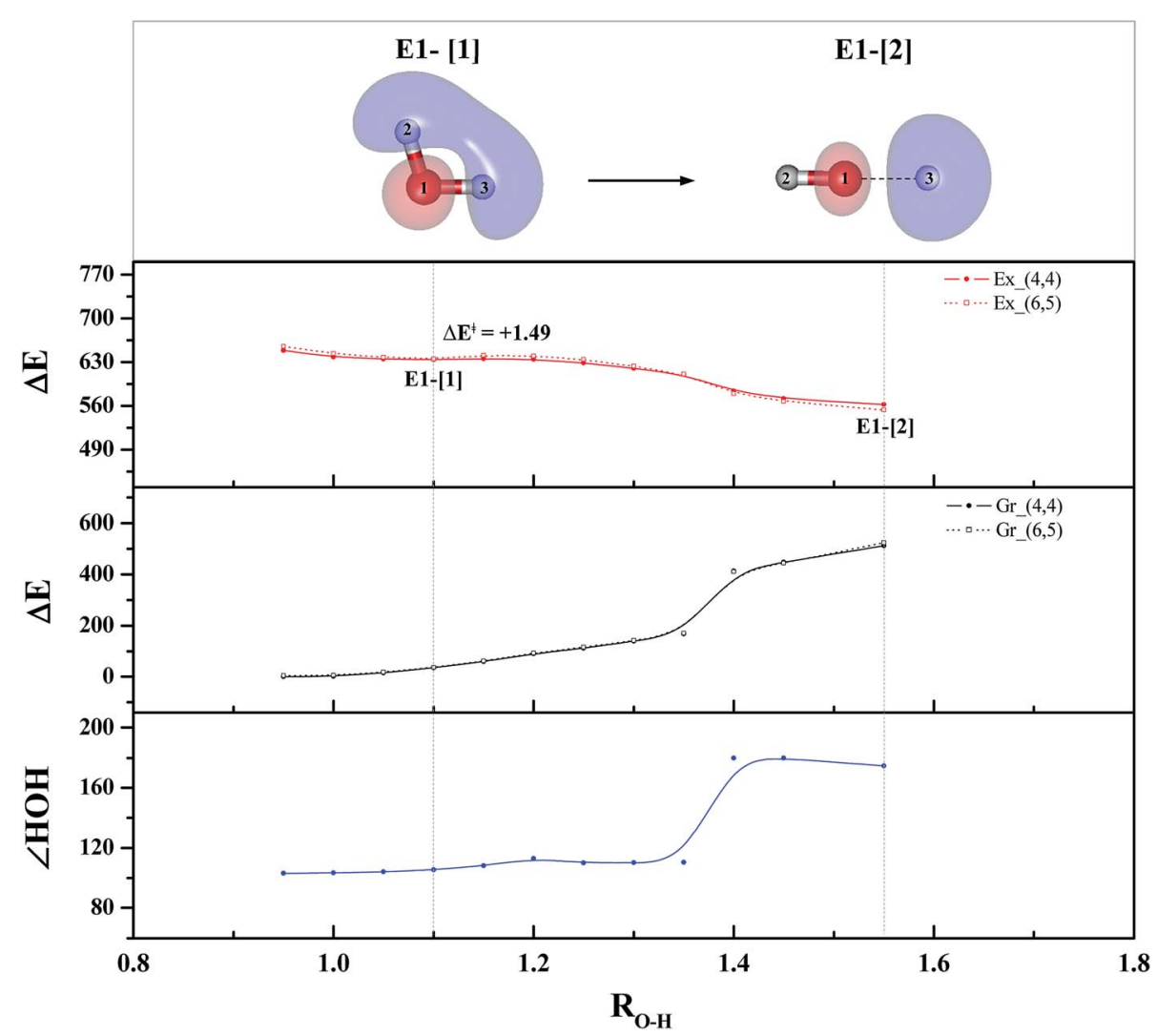

Fig. 3 The $\mathrm{S}_{1}$ relax-scan potential energy curve $\left(\mathrm{Ex}_{-}(4,4)\right)$ with respect to one of the O-H coordinates of the water monomer (structure E1-[2] in Fig. 1) obtained from CASPT2 $(4,4)$ calculations. The energies on the $S_{0}$ potential energy curve $\left(G_{-}(4,4)\right)$ were calculated at the same geometries. The relative energies $(\Delta E)$ are with respect to the total energy of structure G1-[1]. The dash-line curves are the $S_{0}$ and $S_{1}$ energies obtained from CASPT2 $(6,5)$ calculations, Gr_ $(6,5)$ and Ex_(6,5), respectively. The value of the LUMO isosurfaces is 0.065 . Energies, distances and angles are in $\mathrm{kJ} \mathrm{mol}^{-1}, \AA$ and degree, respectively. $\Delta E^{\ddagger}=$ Energy barrier in $\mathrm{kJ} \mathrm{mol}^{-1}$.

electronic ground state is characterized by $R_{\mathrm{O} 1-\mathrm{O} 3}=2.94$ and $R_{\mathrm{O} 1-\mathrm{H} 6}=0.97 \AA(\mathbf{G} 2-[\mathbf{1}])$, which are in excellent agreement with reported theoretical and experimental values, e.g., $R_{\mathrm{O}-\mathrm{O}}=2.93$ and $R_{\mathrm{O}-\mathrm{H}}=0.97 \AA .{ }^{28}$ Moreover, the calculations yield an $\mathrm{S}_{0} \rightarrow \mathrm{S}_{1}$ vertical excitation energy of $E^{\mathrm{Ex}}=7.29 \mathrm{eV}$ for G2-[1], which is comparable to that of the water monomer, $E^{\mathrm{Ex}}=7.20 \mathrm{eV}$ (G1[1]). In the $S_{1}$ state, the CASPT2 $(4,6)$ geometry optimizations of the water dimer in the $C_{1}$ symmetry yielded a transition structure and a global minimum-energy geometry (structures E2-[1] ${ }^{\ddagger}$ and E2-[2] in Fig. 2, respectively). E2-[1] $]^{\ddagger}$ is characterized by an extraordinarily short $\mathrm{H}$-bond $\left(R_{\mathrm{O} 1-\mathrm{O} 3}=2.47 \AA\right)$ and long $\mathrm{O}-\mathrm{H}$ bonds in the water molecule acting as proton donor (H-bond: $R_{\mathrm{O} 1-\mathrm{H} 6}=1.08 \AA$; non-H-bond: $R_{\mathrm{O} 1-\mathrm{H} 2}=1.14 \AA$ ). The short $R_{\mathrm{O} 1-\mathrm{O} 3}$ distance indicates that structure $\mathbf{E 2}-[1]^{\ddagger}$ is characterized by the $\left[\left(\mathrm{H}_{2} \mathrm{O}\right)_{2}\right]^{+}$radical cation or the water dimer cation $\left(R_{\mathrm{O} 1-\mathrm{O} 3}\right.$ $=2.5 \AA),{ }^{29}$ and the long $R_{\mathrm{O} 1-\mathrm{H} 6}$ distance reflects a strong dipoledipole interaction in the $\mathrm{O}-\mathrm{H} \cdots \mathrm{O}$ H-bond $(\mu=4.11 \mathrm{D})$, which suggest the possibility of proton transfer. The long non-H-bond $R_{\mathrm{O} 1-\mathrm{H} 2}$ points to the homolytic cleavage of the $\mathrm{O}_{1}-\mathrm{H}_{2}$ bond and the formation of $[\mathrm{H}]^{*}$, $[\mathrm{OH}]^{*}$, and $\left[\mathrm{H}_{2} \mathrm{O}\right](\mathbf{E} 2-[3])$. Structure $\mathbf{E 2}-[1]^{*}$ could therefore be hypothesized to be the "primary transition structure" for both the photoexcitation and photoionization of the water dimer in the $S_{1}$ state. The existence of structure E2-[1 $]^{\ddagger}$ as the primary transition structure is in accordance with photoelectron-photoion coincident experiments, ${ }^{30}$ in which $\left[\left(\mathrm{H}_{2} \mathrm{O}\right)_{2}\right]^{+\cdot}$ was detected as a minor species with an upper limit of the lifetime of the transient $\left[\mathrm{H}_{2} \mathrm{O}\right]^{+}$radical cation of approximately $40 \mathrm{fs}^{31}$

The global minimum-energy geometry of the water dimer in the $S_{1}$ state (structure E2-[2] in Fig. 2) confirms that the outstanding feature of the $S_{0} \rightarrow S_{1}$ excitation is the proton transfer in H-bond. In addition, based on the spatial distribution of the LUMO, ${ }^{19}$ structure E2-[2] with $R_{\mathrm{O} 1-\mathrm{O} 3}=2.82 \AA$ $\left(\left[\mathrm{H}_{3} \mathrm{O}\right]^{+} \cdots[\mathrm{OH}]^{*}\right)$ can be considered a charge-separated Rydberglike H-bond complex, with the HOMO-LUMO excitation energy of $8.65 \mathrm{eV}$. A similar charge-separated Rydberg-like H-bond complex $\left(\left[\mathrm{NH}_{2} \mathrm{O}\right]^{\cdot} \cdots\left[\mathrm{H}_{3} \mathrm{O}\right]^{+\cdot}\right)$ was confirmed in a previous study to be the smallest, most active intermediate complex for proton transfer in the $\mathrm{NH}_{2} \mathrm{OH} \cdots \mathrm{H}_{2} \mathrm{O}$ system in the $\mathrm{S}_{1}$ state. ${ }^{19}$ These intermediate complexes result from the redistribution of the electron density in the $\mathrm{H}$-bond upon $\mathrm{S}_{0} \rightarrow \mathrm{S}_{1}$ excitation and are represented by electron clouds localized on the two hydrogen atoms of the non-H-bond $\mathrm{O}-\mathrm{H}$ in the $\left[\mathrm{H}_{3} \mathrm{O}\right]^{+\cdot} \cdot{ }^{9,32,33}$ It should be noted that because the CASPT2 $(4,6)$ geometry optimizations were performed without symmetry restrictions (i.e., with $C_{1}$ symmetry), one of the non- $\mathrm{H}$-bond $\mathrm{O}-\mathrm{H}$ distances in the $\left[\mathrm{H}_{3} \mathrm{O}\right]^{+*}$ moiety in structure E2-[2] $\left(R_{\mathrm{O} 3-\mathrm{H} 5}=1.04 \AA\right)$ is slightly longer than the other $\left(R_{\mathrm{O} 3-\mathrm{H} 4}=1.02 \AA\right)$. Therefore, the $\mathrm{O}_{3}-\mathrm{H}_{5}$ 


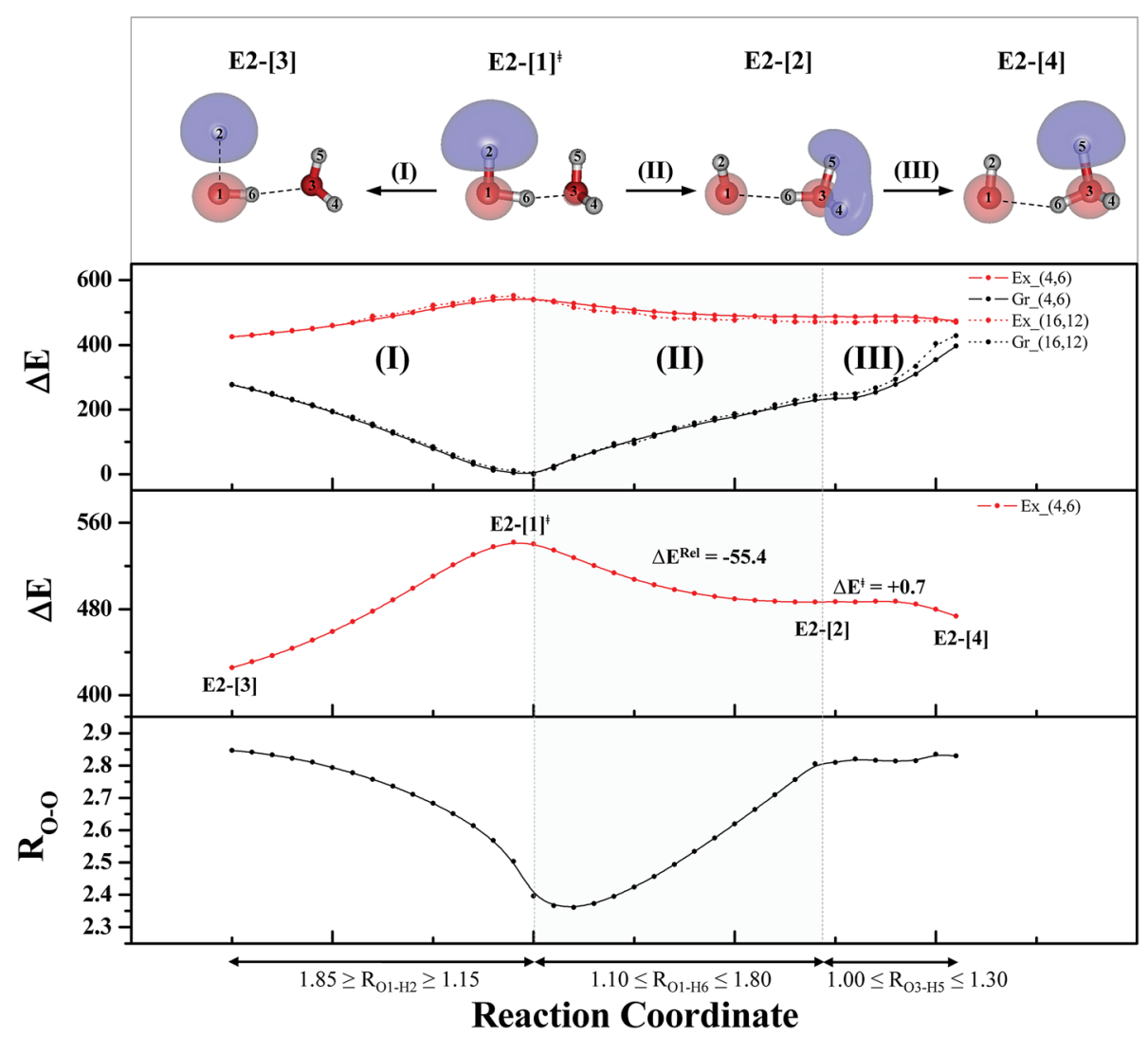

Fig. 4 The $S_{1}$ relax-scan potential energy curves $\left(E_{-}(4,6)\right)$ as a function of the $O-H$ coordinates of the water dimer obtained from CASPT2 $(4,6)$ calculations. The $S_{0}$ potential energy curves $\left(G_{-}(4,6)\right)$ were calculated at the same geometries. The dash-line curves are the $S_{0}$ and $S_{1}$ energies obtained from CASPT2 $(16,12)$ calculations, Gr_ $(16,12)$ and Ex_(16,12), respectively. The relative energies $(\Delta E)$ are with respect to structure G2-[1]. The value of the LUMO isosurfaces is 0.065 . Energies and distances are in $\mathrm{kJ} \mathrm{mol}^{-1}$ and $\AA$, respectively. The energy release ( $\left.\Delta E^{\text {Rel }}\right)$ and energy barrier $\left(\Delta E^{\ddagger}\right)$ are in $\mathrm{kJ} \mathrm{mol}^{-1} ;+=$ transition structure; (I) = photoexcitation; (II) and (III) = photoionization.

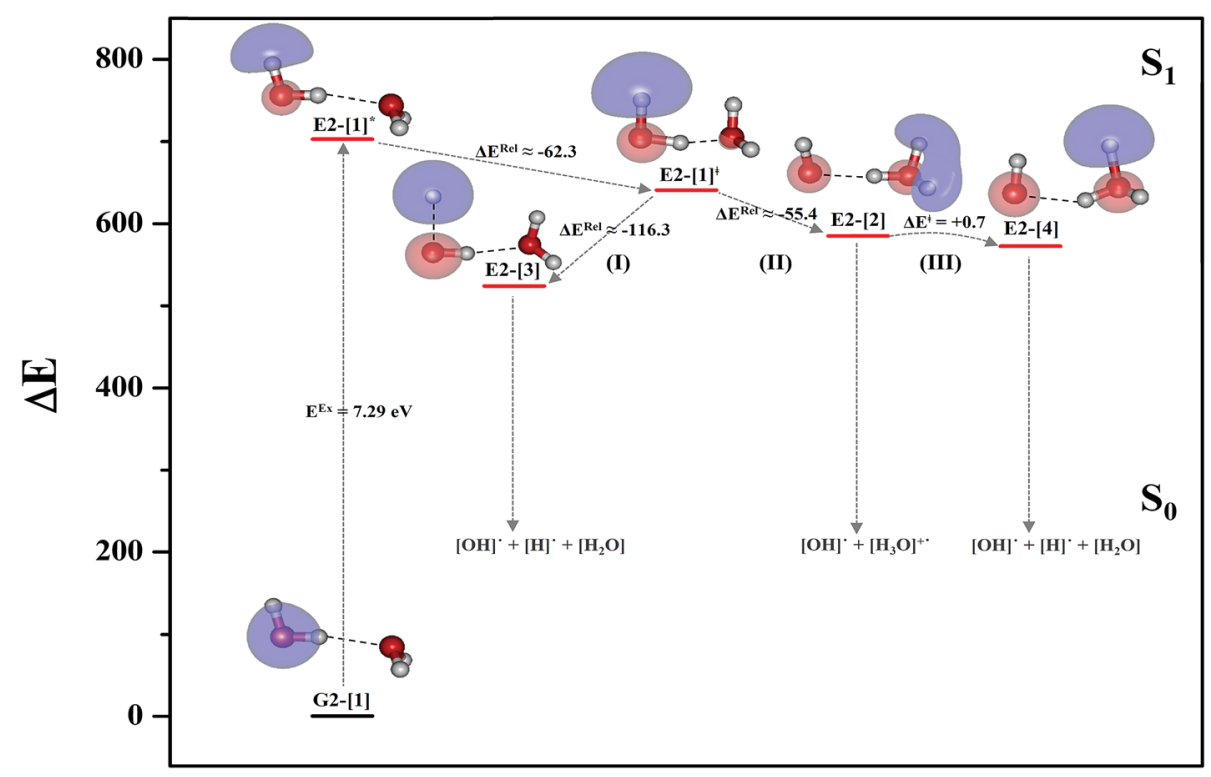

Reaction Paths

Fig. 5 Mechanisms of the photoexcitation ((I)) and photoionization processes ((II) and (III)) of the water dimer in the $\mathrm{S}_{1}$ state obtained from CASPT2 $(4,6)$ calculations. The relative energies $(\Delta E)$ in kJ mol ${ }^{-1}$ are with respect to structure $\mathrm{G} 2-[1]$. The $\mathrm{S}_{0} \rightarrow \mathrm{S}_{1}$ vertical excitation energy $\left(E^{\mathrm{Ex}}\right)$ is in $\mathrm{eV}$. $\Delta E^{\mathrm{Rel}}$ and $\Delta E^{\ddagger}=$ energy release and barrier in $\mathrm{kJ} \mathrm{mol}^{-1}$, respectively; ${ }^{*}=$ vertically excited structure; $\$=$ transition state complex. The value of the LUMO isosurfaces is 0.065 . 

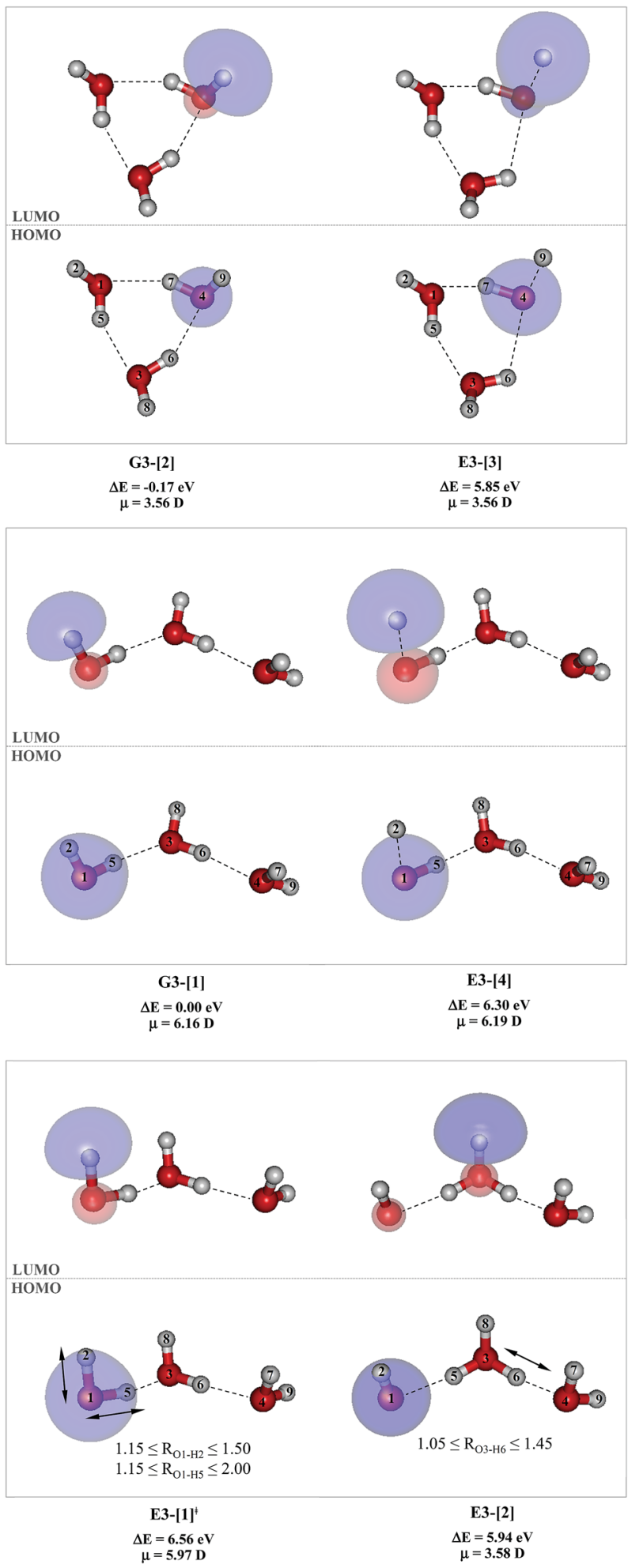

Fig. 6 Structures of the water trimer obtained from CASPT2 $(4,8)$ calculations in the $\mathrm{S}_{0}$ and $\mathrm{S}_{1}$ states; G3-[2] and G3-[1] = global and local minimum energy geometries in the $S_{0}$ state, respectively; E3-[1] $=$ transition structure in the $\mathrm{S}_{1}$ state; $\mathrm{E} 3-[2]=$ equilibrium structure in the $\mathrm{S}_{1}$ state; $\mathrm{E3}-[3]$ and $\mathrm{E3}-[4]=\mathrm{O}-\mathrm{H}$ dissociated structures in the $\mathrm{S}_{1}$ state. $\Delta E=$ Relative energies with respect to the total energy of structure G3-[1]; $\mu=$ dipole moment. The value of the HOMO and LUMO isosurfaces is 0.065 . bond is anticipated to be susceptible to subsequence hydrogen/ proton transfer.

\section{Potential energy curves for the water monomer and dimer}

To study the energetics of the photoexcitation and photoionization processes (eqn (1)-(4)), the $S_{1}$ relax-scan potential energy curves as a function of the $\mathrm{O}-\mathrm{H}$ coordinates of the water monomer and dimer were initially constructed using CASPT2(4,4) and $(4,6)$ calculations and are shown in Fig. 3 and 4, respectively. For the water monomer with the $R_{\mathrm{O} 1-\mathrm{H} 2}$ distance constrained to 1.09 $\AA$, the $\mathrm{S}_{1}$ relax-scan potential energy curve as a function of $R_{\mathrm{O} 1-\mathrm{H} 3}$ exhibits a shallow minimum at $R_{\mathrm{O} 1-\mathrm{H} 3}=1.09 \AA$ (Fig. 3). The homolytic cleavage of the $\mathrm{O}_{1}-\mathrm{H}_{3}$ bond is accompanied by an increase in the $\angle \mathrm{H}_{2} \mathrm{O}_{1} \mathrm{H}_{3}$ from 103 to $180^{\circ}$, with a low energy barrier of $\Delta E^{*}=1.49 \mathrm{~kJ} \mathrm{~mol}^{-1}$ at $R_{\mathrm{O} 1-\mathrm{H} 3}=1.15 \AA$.

Based on the information obtained on the transition and equilibrium structures in Fig. 2, the $\mathrm{S}_{1}$ relax-scan potential energy curves for the photoexcitation and photoionization processes of the water dimer were constructed using CASPT2 $(4,6)$ calculations. Starting from the $\left[\left(\mathrm{H}_{2} \mathrm{O}\right)_{2}\right]^{+\cdot}$ radical cation $\left(\mathbf{E} 2-[1]^{\ddagger}\right)$, the $S_{1}$ relax-scan potential energy curves as a function of the non-H-bond and $\mathrm{H}-b o n d \mathrm{O}-\mathrm{H}$ coordinates were constructed and are shown in Fig. 4 . The $S_{1}$ relax-scan potential energy curve for the photoexcitation in panel (I) is similar to that of the water monomer, which is characterized by a purely repulsive potential energy curve. The outstanding feature of the $S_{1}$ relax-scan potential energy curve for the proton transfer in panel (II) is a shallow potential energy well, corresponding to structure E2-[2] with $R_{\mathrm{O}-\mathrm{O}}=2.80$ and $R_{\mathrm{O}-\mathrm{H}}=1.01 \AA$, and an energy release of $\Delta E^{\mathrm{Rel}} \approx-55.4 \mathrm{~kJ} \mathrm{~mol}^{-1}$ due to the relaxation of E2-[1] $]^{\ddagger}$. The existence of a shallow minimum suggests that the photoionization process is non-concerted, which is supported by the Born-Oppenheimer molecular dynamics (BOMD) results based on the unrestricted MøllerPlesset second-order perturbation theory (UMP2), ${ }^{2}$ in which the ionization process in the ionized water dimer $\left(\left[\left(\mathrm{H}_{2} \mathrm{O}\right)_{2}\right]^{+}\right)$was considerably slower than the photoexcitation process. This finding is different from the results in ref. 9, in which the $S_{1}$ relax-scan potential energy curve obtained from CASPT2 calculations in the $C_{\mathrm{s}}$ symmetry with a larger step length $\left(\Delta R_{\mathrm{O}-\mathrm{H}}=\right.$ $0.20 \AA$ ) is purely repulsive.

Because one of the non-H-bond $\mathrm{O}-\mathrm{H}$ distances in the $\left[\mathrm{H}_{3} \mathrm{O}\right]^{+}$. moiety of structure E2-[2] is longer than the other, the possibility of the hydrogen/proton transfer from $\left[\mathrm{H}_{3} \mathrm{O}\right]^{+}$was investigated. The $\mathrm{S}_{1}$ relax-scan potential energy curve for the $\mathrm{O}_{3}-\mathrm{H}_{5}$ dissociation was tentatively constructed and is included in Fig. 4, panel (III). The potential energy curve indicates that the extension of the $\mathrm{O}_{3}-\mathrm{H}_{5}$ bond leads to structure E2-[4], with a small energy barrier of $\Delta E^{\ddagger}=0.7 \mathrm{~kJ} \mathrm{~mol}^{-1}$ for the $\mathrm{O}_{3}-\mathrm{H}_{5}$ dissociation. Because the $S_{1}$ relax-scan potential energy curve starts to decline at $R_{\mathrm{O} 3-\mathrm{H} 5}=1.30 \AA$ and $E^{\mathrm{Ex}}=0.79 \mathrm{eV}$, the intersection of the $\mathrm{S}_{0}$ and $\mathrm{S}_{1}$ states, which generates $[\mathrm{H}]^{\circ},[\mathrm{OH}]^{\circ}$, and $\left[\mathrm{H}_{2} \mathrm{O}\right]$ in their respective electronic ground states, is anticipated to occur beyond this point.

Based on the equilibrium and transition structures as well as on the $S_{1}$ relax-scan potential energy curves obtained from 


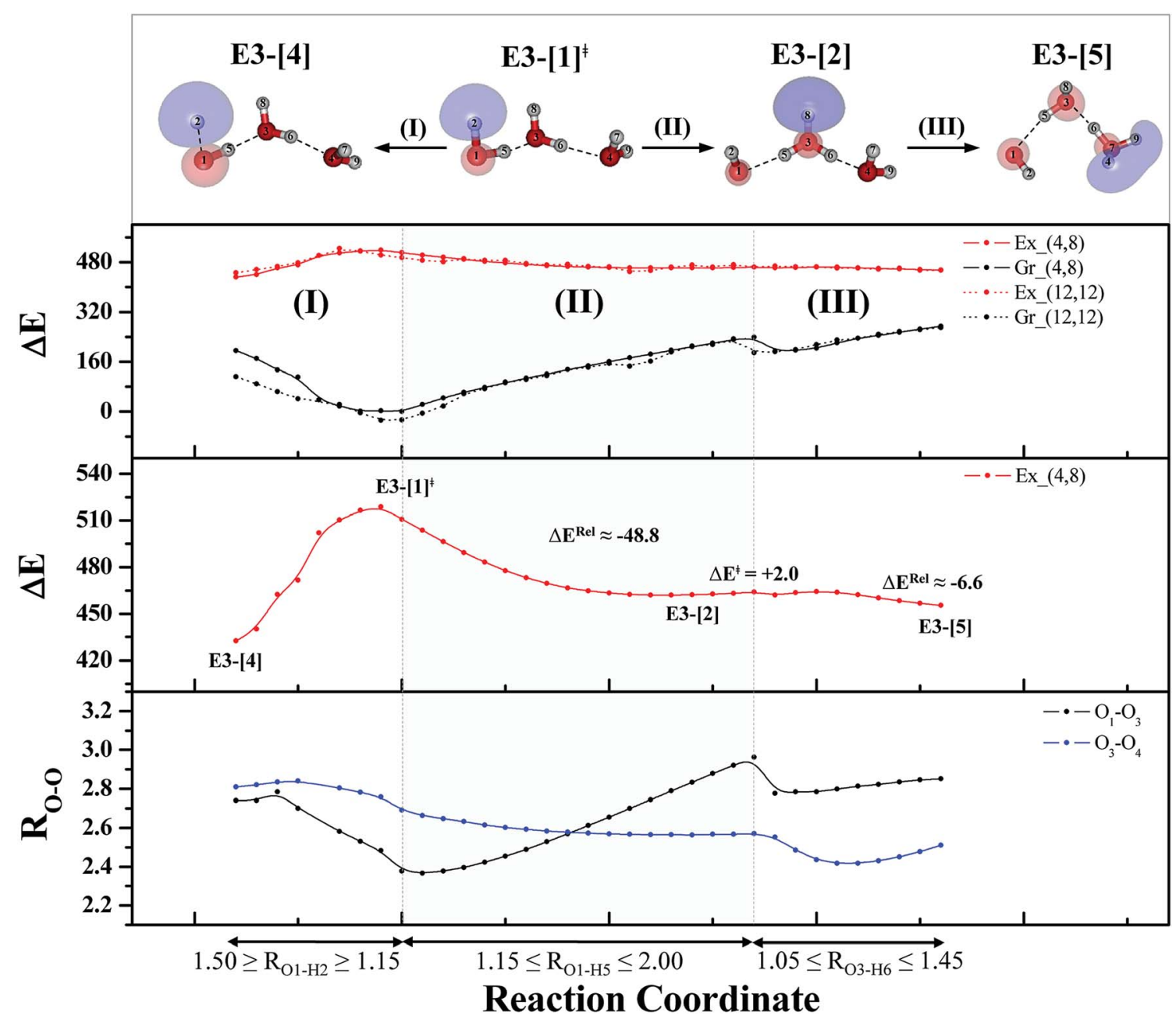

Fig. 7 The $\mathrm{S}_{1}$ relax-scan potential energy curves $\left(\mathrm{Ex}_{-}(4,8)\right)$ as a function of the $\mathrm{O}-\mathrm{H}$ coordinates of the water trimer obtained from $\mathrm{CASPT} 2(4,8)$ calculations. The $S_{0}$ potential energy curves $\left(G_{-}(4,8)\right)$ were calculated at the same geometries. The dash-line curves are the $S_{0}$ and $S_{1}$ energies obtained from CASPT2 $(12,12)$ calculations, Gr_(12,12) and Ex_(12,12), respectively. The relative energies $(\Delta E)$ are with respect to structure G3-[1]. The value of the LUMO isosurfaces is 0.065 . Energies and distances are in $\mathrm{kJ} \mathrm{mol}^{-1}$ and $\AA$, respectively. The energy release $\left(\Delta E^{\mathrm{Rel}}\right)$ and energy barrier $\left(\Delta E^{\ddagger}\right)$ are in $\mathrm{kJ} \mathrm{mol}^{-1} ; *=$ transition structure; (I) = photoexcitation; (II) and (III) = photoionization.

CASPT2 $(4,6)$ calculations, the photoexcitation and photoionization mechanisms in the water dimer can be hypothesized to occur through the primary transition structure $\left(\mathbf{E} 2-[\mathbf{1}]^{\ddagger}\right)$, as shown in Fig. 5. Starting from the ground-state geometry (G2$[1])$, the $S_{0} \rightarrow S_{1}$ vertical excitation $\left(E^{\mathrm{Ex}}=7.29 \mathrm{eV}\right)$ leads to structure E2-[1]*, which is relaxed to the $\left[\left(\mathrm{H}_{2} \mathrm{O}\right)_{2}\right]^{+\cdot}$ radical cation $\left(\mathbf{E 2}-[\mathbf{1}]^{*}\right)$. Structure $\mathbf{E 2}-[\mathbf{1}]^{*}$ generates $[\mathrm{H}]^{*},[\mathrm{OH}]^{\circ}$, and $\left[\mathrm{H}_{2} \mathrm{O}\right]$ in their respective electronic-ground states either directly through photoexcitation (I) or indirectly through the metastable $\left[\mathrm{H}_{3} \mathrm{O}\right]^{+} \cdot \ldots[\mathrm{OH}]^{\bullet}$ Rydberg-like H-bond complex (structure E2-[2]) via $\mathrm{O}_{3}-\mathrm{H}_{5}$ dissociation from $\left[\mathrm{H}_{3} \mathrm{O}\right]^{+}$, as seen in panel (II) and panel (III), respectively. The hypothesized mechanisms imply that the photoexcitation process in water clusters is not necessarily unimolecular as proposed in eqn (1) and (2), and the $\left[\left(\mathrm{H}_{2} \mathrm{O}\right)_{2}\right]^{+\cdot}$ radical cation can be considered the smallest intermediate complex in the photoionization process (eqn (3) and (4)). The proposed water dimer as a precursor for the photodissociation processes is supported by a submillimeter-wave absorption spectrum of the Earth's atmosphere and experiments, ${ }^{34,35}$ which suggested that the absorption results from the water dimer existing at low altitude in the troposphere and lower stratosphere.

\section{Equilibrium structures and potential energy curves for the water trimer}

To confirm the mechanisms proposed in Fig. 5, CASPT2 $(4,8)$ calculations were performed on the water trimer, and the characteristic structures are shown with the spatial distributions of the HOMO and LUMO in Fig. 6. In the electronicground state, the global minimum-energy geometry of the water trimer is represented by a cyclic H-bonded structure (G3[2]) with three equivalent $\mathrm{O}-\mathrm{H} \cdots \mathrm{O} H$-bonds $\left(R_{\mathrm{O}-\mathrm{O}}=2.81 \AA\right)$ and a $\mathrm{S}_{0} \rightarrow \mathrm{S}_{1}$ vertical excitation energy of $E^{\mathrm{Ex}}=7.62 \mathrm{eV}(163 \mathrm{~nm})$, whereas the local minimum-energy geometry is a linear $\mathrm{H}$ bonded structure (G3-[1]) with $R_{\mathrm{O}-\mathrm{O}}=2.87$ and $2.92 \AA, R_{\mathrm{O}-\mathrm{H}}=$ 0.96-0.98 $\AA$, and $E^{\mathrm{Ex}}=7.17 \mathrm{eV}(173 \mathrm{~nm})$. Because the $\mathrm{S}_{0} \rightarrow \mathrm{S}_{1}$ vertical excitation energy is almost the same as that of the water 


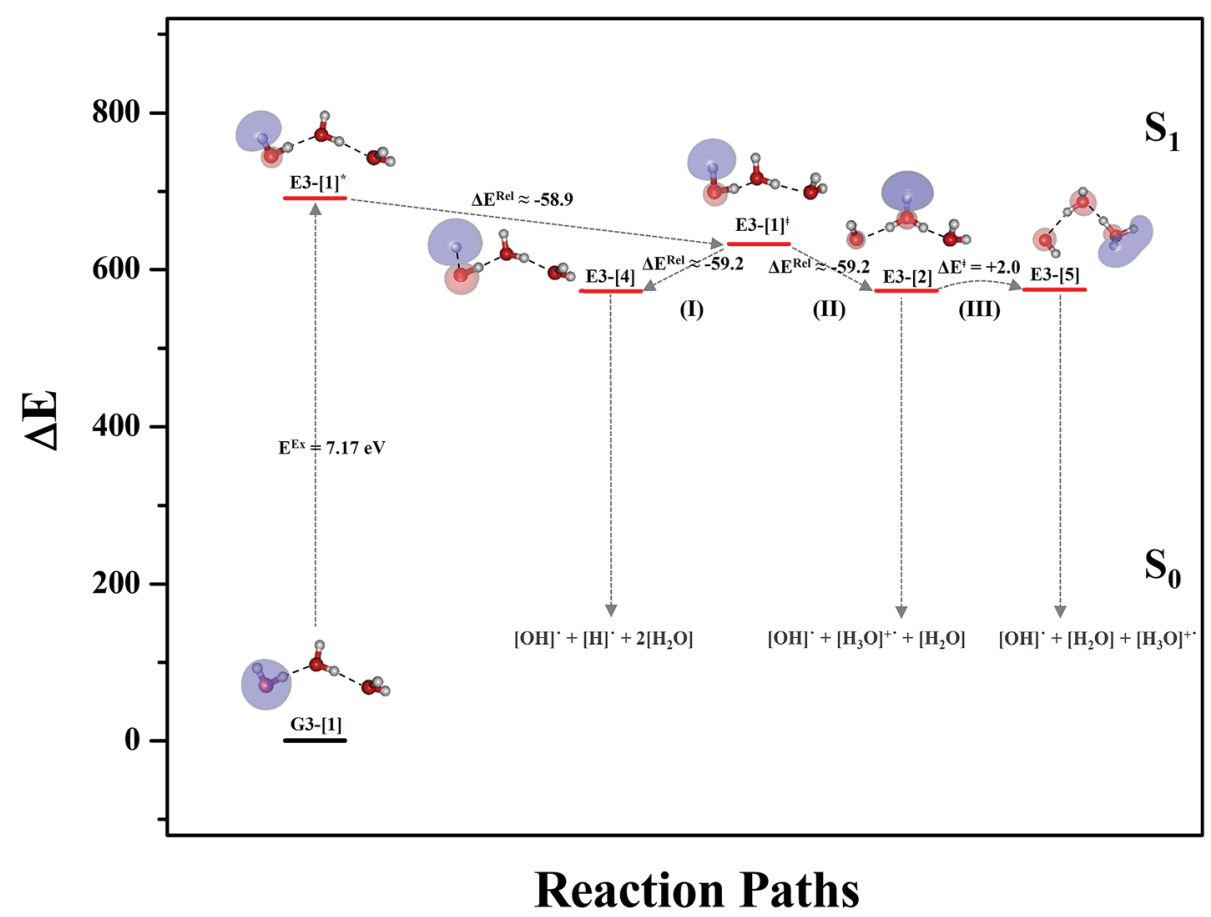

Fig. 8 Mechanisms of the photoexcitation ((I)) and photoionization processes ((II) and (III)) of the water trimer in the $S_{1}$ state obtained from CASPT2 $(4,8)$ calculations. The relative energies $(\Delta E)$ are with respect to structure G3-[1] and in $\mathrm{kJ} \mathrm{mol}^{-1}$. The $\mathrm{S}_{0} \rightarrow \mathrm{S}_{1}$ vertical excitation energy $\left(E^{\mathrm{Ex}}\right)$ is in $\mathrm{eV} . \Delta E^{\mathrm{Rel}}$ and $\Delta E^{*}=$ energy release and barrier in $\mathrm{kJ} \mathrm{mol}^{-1}$, respectively; ${ }^{*}=$ vertically excited structure; $\$=$ transition state complex. The value of the LUMO isosurfaces is 0.065 .

monomer and dimer (7.20 and $7.29 \mathrm{eV}$, respectively), the energy of a single photon will excite only one oxygen atom in the water clusters; for the water dimer and trimer, the spatial distributions of the HOMO and LUMO in Fig. 2 and 6 suggest that a single photon vertically excites the oxygen lone-pair of the water molecule acting as proton donor, e.g., structures G2-[1] and G3-[1], and the lone-pairs and Rydberg orbitals of all of the oxygen atoms in the small water clusters are most likely not degenerate due to the $\mathrm{H}$-bond formation.

Because the linear H-bonded trimer (E3-[1] $\left.]^{*}\right)$ is energetically more stable (approximately $29 \mathrm{~kJ} \mathrm{~mol}^{-1}$ ) than the cyclic $\mathrm{H}$ bonded trimer (E3-[2]*), only the linear $\mathrm{H}$-bond trimer was used as a representative system in the study of the effects of the cluster size. Starting from the transition structure E2-[1] ${ }^{\ddagger}$ with an additional water molecule, CASPT2 geometry optimizations yield structure E3-[1 $]^{\ddagger}$, in which the $\left[\left(\mathrm{H}_{2} \mathrm{O}\right)_{2}\right]^{+\cdot}$ radical cation moiety is approximately the same as in structure E2-[1 $]^{\ddagger}\left(\mathbf{E} 3-[\mathbf{1}]^{+}\right.$; $\left.R_{\mathrm{O} 1-\mathrm{O} 3}=2.47, R_{\mathrm{O} 1-\mathrm{H} 2}=1.13, R_{\mathrm{O} 1-\mathrm{H} 5}=1.10 \AA, \mu=5.97 \mathrm{D}\right)$. Similarly, starting from the intermediate structure E2-[2] with one water molecule $\mathrm{H}$-bonded to $\left[\mathrm{H}_{3} \mathrm{O}\right]^{+\cdot}$, structure E3-[2] is obtained as the global minimum-energy geometry with a subunit resembling the $\left[\mathrm{H}_{3} \mathrm{O}\right]^{+} \ldots[\mathrm{OH}]^{*}$ charge-separated Rydberg-like dimer (E3-[2]; $R_{\mathrm{O} 1-\mathrm{O} 3}=2.78$ and $R_{\mathrm{O} 3-\mathrm{O} 4}=2.59$ $\AA$ ). These findings confirm that structures E2-[1] $]^{\ddagger}$ and E2-[2] are the smallest, most active transition and intermediate complexes for the photoionization process, and that proton transfer from the intermediate structure E2-[2] is possible owing to the $\mathrm{H}^{-}$ bond chain extension.
Comparison of the shapes of the $\mathrm{S}_{1}$ relax-scan potential energy curves and the spatial distributions of the LUMO as a function of the $\mathrm{O}-\mathrm{H}$ coordinates in Fig. 4 and 7 shows no significant differences between the photoexcitation and photoionization processes in the water dimer and trimer. Owing to the H-bond chain extension, the $\left[\mathrm{H}_{3} \mathrm{O}\right]^{+} \ldots[\mathrm{OH}]^{\circ}$ charge-separated Rydberg-like moiety is stabilized, which is reflected in the high energy barrier for proton transfer in panel (III) $\left(\Delta E^{\ddagger}=2.0 \mathrm{~kJ} \mathrm{~mol}^{-1}\right)$ compared to that of structure $\mathbf{E 2}$-[2] $\left(\Delta E^{\ddagger}=0.70 \mathrm{~kJ} \mathrm{~mol}^{-1}\right)$. Based on the information obtained for the water trimer, the relative energies for the photoexcitation and photoionization mechanisms, in which the $\left[\left(\mathrm{H}_{2} \mathrm{O}\right)_{2}\right]^{+*}$ radical cation moiety being the primary transition structure, are illustrated in Fig. 8. These structural and energetic results for the water trimer confirm that the extension of the H-bond chain from $n=2$ to 3 does not lead to significant changes in the transition and intermediate complexes, and the water dimer is the smallest transition structure in the photoionization process of water clusters in gas phase.

\section{Analyses of electronic configurations and the CI coefficients}

To obtain information on the electronic configurations and multireference characters in the photodissociation processes, the main electronic configurations and CI coefficients (cut-off $=$ 0.05 ) of the water monomer, dimer and trimer in the $\mathrm{S}_{0}$ and $\mathrm{S}_{1}$ states, obtained from CASPT2 calculations with restricted and extended complete active spaces, are included in Tables 2-4, respectively; $\Psi_{0}$ and $C_{0}=$ ground state determinant and CI 
coefficient; $\Psi_{a}^{r}$ and $C_{a}^{r}=a \rightarrow r$ singly excited determinant (Stype) and CI coefficient and; $\Psi_{a, b}^{r, s}$ and $C_{a, b}^{r, s}=a \rightarrow r$ and $b \rightarrow s$ doubly excited determinant (D-type) and CI coefficient; the indices $a$ and $b$, and $r$ and $s$ label occupied and virtual or unoccupied spin orbitals, respectively; a bar or lack of a bar is to denote beta $(\beta)$ and alpha $(\alpha)$ spin orbitals, respectively.

In the $S_{1}$ state, the analyses of the electronic configurations and CI coefficients obtained from CASPT2 calculations with restricted and extended complete active spaces show a common trend for the small water clusters. For example, for the water dimer, CASPT2 $(4,6)$ calculations in Table 3 suggest that singly excited determinants $(\Psi \overline{11})$ dominate doubly excited determinants for the equilibrium (E2-[2]; $C_{\overline{10}}^{\overline{11}}=0.9739$; $\left.C_{9, \overline{10}}^{11, \overline{11}}=0.1231\right)$ and transition structures $\left(\mathbf{E} 2-[1]^{*} ; C_{\overline{10}}^{\overline{11}}=0.9779\right.$; $\left.C_{10, \overline{10}}^{11, \overline{13}}=0.0859\right)$, whereas for the $\mathrm{O}-\mathrm{H}$ dissociated structure, the contribution of the singly excited determinant $\left(\Psi \frac{\overline{11}}{10}\right)$ decreases and that of the doubly excited determinant $\left(\Psi_{9, \overline{10}}^{11, \overline{11}}\right)$ increases (E2-[3]; $C_{\overline{10}}^{\overline{11}}=0.9409$ and $\left.C_{9, \overline{10}}^{11, \overline{11}}=0.3193\right)$.

CASPT2 calculations with restricted and extended complete active spaces also show the dependence of the CI coefficients for the $\mathrm{O}-\mathrm{H}$ dissociated structure in the $\mathrm{S}_{0}$ state. For example, for the water dimer, CASPT2 $(4,6)$ calculations illustrate that the ground state configuration $\left(\Psi_{0}\right)$ is outstanding for the equilibrium structure (G2-[1]; $C_{0}=0.9845$ ), and upon the O-H dissociation, the contribution of $\Psi_{0}$ decreases, whereas that of the singly excited determinant $\left(\Psi_{\overline{9}}^{\overline{11}}\right)$ increases $\left(C_{0}=0.8477\right.$; $\left.C_{\overline{9}}^{\overline{11}}=0.4003\right)$. The discussion in this subsection leads to the conclusion that the multireference characters of the wave functions are important in both $\mathrm{S}_{0}$ and $\mathrm{S}_{1}$ states, especially for the $\mathrm{O}-\mathrm{H}$ dissociated structures, and are not significantly changed with the number of water molecules.

\section{Conclusion}

Herein, the mechanisms of photoexcitation and photoionization of small water clusters in gas phase, $\left(\mathrm{H}_{2} \mathrm{O}\right)_{n} ; n=2-3$, were studied. In contrast to the previous theoretical studies, which considered the photoexcitation and photoionization processes separately, this study characterized the structures and energetics of common transition and intermediate complexes in the lowest singlet-excited $\left(S_{1}\right)$ state and the effects of the cluster size. These objectives were achieved by performing CASPT2 geometry optimizations and transition state calculations without symmetry constraints ( $C_{1}$ symmetry) and by allowing all the degrees of freedom to freely relax during the construction of the $S_{1}$ potential energy curves.

The $S_{1}$ relax-scan potential energy curves as a function of the $\mathrm{O}-\mathrm{H}$ coordinates revealed that the photoexcitation of the water monomer by a single photon can directly generate $[\mathrm{OH}]^{\circ}$ and $[\mathrm{H}]^{\cdot}$ in their respective electronic-ground states, and a single photon with approximately the same energy can similarly lead to the photoexcitation and also to the photoionization in the water clusters. The $\mathrm{S}_{0} \rightarrow \mathrm{S}_{1}$ excitation of the water clusters results in strong polarization in the $\mathrm{O}-\mathrm{H} \cdots \mathrm{O} \mathrm{H}$-bond and thus in the formation of the primary transition structure for both photoexcitation and photoionization, which implies that the photoionization process can be activated by photon with the energy lower than the ionization threshold of water molecule $(12.6 \mathrm{eV})$. This primary transition structure is characterized by the water dimer radical cation $\left(\left[\left(\mathrm{H}_{2} \mathrm{O}\right)_{2}\right]^{+}\right)$with $C_{1}$ symmetry, from which $[\mathrm{OH}]^{*},[\mathrm{H}]^{\cdot}$, and $\left[\mathrm{H}_{3} \mathrm{O}\right]^{+*}$ can be generated through the homolytic cleavage of an $\mathrm{O}-\mathrm{H}$ bond and through proton transfer to form the metastable charge-separated Rydberg-like H-bond complex $\left(\left[\mathrm{H}_{3} \mathrm{O}\right]^{+} \cdots[\mathrm{OH}]^{*}\right)$ upon photoexcitation and photoionization, respectively. These results are different from those of previously reported $a b$ initio calculations in the $C_{\mathrm{s}}$ point group, in which the photoionization in the $S_{1}$ state was concluded to take place on a purely repulsive potential energy curve.

The results on the water trimer confirmed these findings and further concluded that the $\left[\left(\mathrm{H}_{2} \mathrm{O}\right)_{2}\right]^{+\cdot}$ and $\left[\mathrm{H}_{3} \mathrm{O}\right]^{+} \ldots[\mathrm{OH}]^{\cdot} \mathrm{H}^{-}$ bonded complexes are the smallest transition and intermediate complexes in the photoionization process in the $\mathrm{S}_{1}$ state; the ab initio results showed that increasing the size of the water clusters does not lead to significant changes in the structures and energetics of these charged species. The mechanisms proposed in this work further suggest that the photoexcitation and photoionization processes in gas phase are most likely bimolecular reactions, in which the transition and intermediate charged species are more stabilized than in a unimolecular reaction. These theoretical results provide insights into the photoexcitation and photoionization mechanisms of molecular clusters and can be used as guidelines for further theoretical and experimental studies.

\section{Conflicts of interest}

There are no conflicts to declare.

\section{Acknowledgements}

The authors would like to acknowledge the financial support provided by SUT Research and Development Fund, Project Number: IRD1-102-58-12-07.

\section{References}

1 D. David, I. Bar and S. Rosenwaks, J. Phys. Chem., 1993, 97, 11571-11574.

2 A. Kumar, M. Kołaski, H. M. Lee and K. S. Kim, J. Phys. Chem. A, 2008, 112, 5502-5508.

3 J. M. Heller Jr, R. N. Hamm, R. D. Birkhoff and L. R. Painter, J. Chem. Phys., 1974, 60, 3483.

4 W. A. Goddard III and W. J. Hunt, Chem. Phys. Lett., 1974, 24, 457-628.

5 M. Robin, Higher Excited States of Polyatomic Molecules, Academic Press, New York, 1985.

6 R. Mota, R. Parafita, A. Giuliani, M.-J. Hubin-Franskin, J. M. C. Lourenço, G. Garcia, S. V. Hoffmann, N. J. Mason, P. A. Ribeiro, M. Raposo and P. Limão-Vieira, Chem. Phys. Lett., 2005, 416, 152-159. 
7 M. Rubio, L. Serrano-Andrés and M. Merchán, J. Chem. Phys., 2008, 128, 104305.

8 W. R. Wadt and W. A. Goddard III, Chem. Phys., 1976, 18, 111.

9 A. L. Sobolewski and W. Domcke, Phys. Chem. Chem. Phys., 2002, 4, 4-10.

10 M. Kowal, S. Roszak and J. Leszczynski, J. Chem. Phys., 2001, 114, 8251.

11 H. Tachikawa, Phys. Chem. Chem. Phys., 2011, 13, 1120611212.

12 N. Agmon, Isr. J. Chem., 1999, 39, 493-502.

13 T. C. Berkelbach, H.-S. Lee and M. E. Tuckerman, Phys. Rev. Lett., 2009, 103, 238302.

14 D. Marx, A. Chandra and M. E. Tuckerman, Chem. Rev., 2010, 110, 2174-2216.

15 P. Suwannakham and K. Sagarik, $R S C A d v .$, 2017, 7, 2149221506.

16 C. Lao-ngam, P. Asawakun, S. Wannarat and K. Sagarik, Phys. Chem. Chem. Phys., 2011, 13, 4562.

17 C. Lao-ngam, M. Phonyiem, S. Chaiwongwattana, Y. Kawazoe and K. Sagarik, Chem. Phys., 2013, 420, 50-61.

18 K. Sagarik, S. Chaiwongwattana, V. Vchirawongkwin and S. Prueksaaroon, Phys. Chem. Chem. Phys., 2010, 12, 918.

19 J. Thisuwan, P. Suwannakham, C. Lao-ngam and K. Sagarik, Phys. Chem. Chem. Phys., 2016, 18, 5564-5579.

20 L. Gonzalez, D. Escudero and L. Serrano-Andres, ChemPhysChem, 2012, 13, 28-51.

21 M. Schreiber, M. R. Silva-Junior, S. P. A. Sauer and W. Thiel, J. Chem. Phys., 2008, 128, 134110.

22 H.-J. Werner, P. J. Knowles, G. Knizia, F. R. Manby and M. Schütz, WIREs Comput Mol Sci, 2012, vol. 2, pp. 242-253. 23 H.-J. Werner, P. J. Knowles, G. Knizia, F. R. Manby, M. Schütz, P. Celani, T. Korona, R. Lindh, A. Mitrushenkov, G. Rauhut, K. R. Shamasundar,
T. B. Adler, R. D. Amos, A. Bernhardsson, A. Berning, D. L. Cooper, M. J. O. Deegan, A. J. Dobbyn, F. Eckert, E. Goll, C. Hampel, A. Hesselmann, G. Hetzer, T. Hrenar, G. Jansen, C. Köppl, Y. Liu, A. W. Lloyd, R. A. Mata, A. J. May, S. J. McNicholas, W. Meyer, M. E. Mura, A. Nicklaß, D. P. O'Neill, P. Palmieri, D. Peng, K. Pflüger, R. Pitzer, M. Reiher, T. Shiozaki, H. Stoll, A. J. Stone, R. Tarroni, T. Thorsteinsson and M. Wang, MOLPRO, a package of ab initio programs, 2015, available from http:// www.molpro.net.

24 P. J. Knowles and H.-J. Werner, Chem. Phys. Lett., 1985, 115, 259-267.

25 H.-J. Werner and P. J. Knowles, J. Chem. Phys., 1985, 82, 5053-5063.

26 H.-J. Werner and W. Meyer, J. Chem. Phys., 1980, 73, 23422356.

27 J. Q. Sun and K. Ruedenberg, J. Chem. Phys., 1994, 101, 2157. 28 K. S. Kim, B. J. Mhin, U. S. Choi and K. Lee, J. Chem. Phys., 1992, 97, 6649.

29 P. A. Pieniazek, J. VandeVondele, P. Jungwirth, A. I. Krylov and S. E. Bradforth, J. Phys. Chem. A, 2008, 112, 6159-6170.

30 K. Norwood, A. Ali and C. Y. Ng, J. Chem. Phys., 1991, 95, 8029.

31 O. Marsalek, C. G. Elles, P. A. Pieniazek, E. Pluhařová, J. VandeVondele, S. E. Bradforth and P. Jungwirth, J. Chem. Phys., 2011, 135, 224510.

32 A. L. Sobolewski and W. Domcke, J. Phys. Chem. A, 2002, 106, 4158-4167.

33 A. L. Sobolewski and W. Domcke, Phys. Chem. Chem. Phys., 2007, 9, 3818-3829.

34 H. A. Gebbie, W. J. Burroughs, J. Chamberlain, J. E. Harries and R. G. Jones, Nature, 1969, 221, 143-145.

35 B. Ruscic, J. Phys. Chem. A, 2013, 117, 11940-11953. 